\title{
CONTROL OF NETWORKS OF EULER-BERNOULLI BEAMS
}

\author{
Bertrand DEKONINCK ${ }^{1}$ AND SERGE NiCAISE ${ }^{1}$
}

\begin{abstract}
We consider the exact controllability problem by boundary action of hyperbolic systems of networks of Euler-Bernoulli beams. Using the multiplier method and Ingham's inequality, we give sufficient conditions insuring the exact controllability for all time. These conditions are related to the spectral behaviour of the associated operator and are sufficiently concrete in order to be able to check them on particular networks as illustrated on simple examples.

Résumé. Nous considérons le problème de la contrôlabilité exacte par contrôle frontière du système hyperbolique des poutres d'Euler-Bernoulli sur des réseaux. Utilisant la méthode des multiplicateurs et les inégalités d'Ingham, nous donnons des conditions suffisantes qui assurent la contrôlabilité exacte pour tout temps. Ces conditions sont relatives au comportement spectral de l'opérateur associé et sont suffisamment explicites de sorte qu'elles peuvent être vérifiée pour un réseau donné comme illustré sur des exemples simples.
\end{abstract}

AMS Subject Classification. 93C20 35B37 35P20.

Received February 24, 1998. Revised September 2, 1998.

\section{INTRODUCTION}

The description of various models of multiple-link flexible structures, consisting of finitely many interconnected flexible elements, like strings, beams, plates, shells or combinations of them, recently has a great interest $[8-10,13,21,23,24]$. The problem of controllability or stabilizabilition of such structures is an expanding field. For control results, let us quote the works of Lagnese-Leugering-Schmidt $[22,23,25,32]$ for 1-d networks; the works of Puel and Zuazua [31], Lagnese [20] and the second author [27-30] for multidimensional structures. For stabilization results, we may cite the papers of Chen et al. [10-12] and of Conrad [14]. In the above papers about control problems except [25], the hyperbolic system is of wave type and is then characterized by a finite speed of propagation, as a consequence there exists a minimal positive time (depending on the geometry of the domain) to have exact controllability. In the present paper, we consider Petrovsky systems on 1-d networks and we show how to manage the network structure and the controllability problem using the Hilbert Uniqueness Method (HUM) of Lions [26]. Since the multiplier method is relatively limited and only allows to show the so-called inverse inequality for star-shaped networks (as in [25]), we have decided to give sufficient conditions insuring the exact controllability for all time $T>0$ with the help of HUM, but sufficiently explicit in order to check them in practice. We further show that one of these conditions is also necessary. At the end our results are illustrated by some simple examples.

\footnotetext{
Keywords and phrases: Control, Euler-Bernoulli beams, networks, spectral analysis

${ }^{1}$ Université de Valenciennes et du Hainaut Cambrésis, LIMAV, BP. 311, 59304 Valenciennes Cedex, France;

e-mail: snicaise@univ-valenciennes.fr
} 
In the physical point of view, the model considered in this paper is of interest in the collinear case. The noncollinear case is a first step for the study of more realistic vectorial models, like in-plane or 3-d beam structures as considered in $[23,25]$. Actually the boundary conditions at the multiple joints (called transmission conditions) were chosen so that for two beams joints they reduce to the usual boundary conditions for two collinear beams. Note that our results directly extend to other kinds of transmission conditions like those from $[9,15]$. We believe that the vectorial models may be handled in a similar way.

The schedule of the paper is the following one: in Section 2, we recall some notations and definitions concerning 1-d networks and introduce the (spatial) operator, namely a fourth order operator on each edge with some transmission conditions at interior nodes and clamped boundary conditions at exterior nodes. Section 3 is devoted to the solution of the associated Petrovsky system and of the proof of the direct inequality using the usual multiplier method. In Section 4, we establish the inverse inequality for star-shaped networks, first for $T$ large enough by the multiplier techniques and secondly for all $T>0$ using the results from ([19] Chap. 5). This yields the equivalence between the energy and the $L^{2}$-norm on the (external) lateral boundary of the second derivative of the solution of the Petrovsky system. Since the multiplier method only works for star-shaped networks and since we want to consider other networks, we give sufficient conditions insuring with the help of Ingham's inequality that the above $L^{2}$-norm is a norm on the space of initial data for all time $T>0$. These conditions are related to the spectral properties of the spatial operator and then may be checked for a given network. The weak solution of the Petrovsky system is considered in Section 5 as well as its interpretation in terms of partial differential equations. The Hilbert Uniqueness Method is presented in Section 6 . Finally, in Section 7 we show that one of the above sufficient conditions to have exact controllability is also necessary and we give some examples: some where we have exact controllability by checking the sufficient conditions mentioned above and one for which we do not have exact controllability (by exterior boundary control). As in ([23], § II.5.2), for this counterexample we have chosen a network with a circuit because we conjecture that all networks with (at least) one circuit are not exactly controllable.

\section{Preliminaries}

We first recall the notion of $C^{\nu}$-networks, $\nu \in \mathbb{N}=\{0,1,2, \ldots\}$, which is simply those of [5], we refer to $[1,4,6,7]$ for more details.

All graphs considered here are non empty, finite and simple. Let $\Gamma$ be a connected topological graph imbedded in $\mathbb{R}^{m}, m \in \mathbb{N}^{*}=\mathbb{N} \backslash\{0\}$, with $n$ vertices and $N$ edges. Let $E=\left\{E_{i}: 1 \leq i \leq n\right\}\left(\right.$ resp. $\left.K=\left\{k_{j}: 1 \leq j \leq N\right\}\right)$ be the set of vertices (resp. edges) of $\Gamma$. Each edge $k_{j}$ is a Jordan curve in $\mathbb{R}^{m}$ and is assumed to be parametrized by its arc length parameter $x_{j}$, such that the parametrizations

$$
\pi_{j}:\left[0, l_{j}\right] \rightarrow k_{j}: x_{j} \mapsto \pi_{j}\left(x_{j}\right)
$$

is $\nu$-times differentiable, i.e., $\pi_{j} \in C^{\nu}\left(\left[0, l_{j}\right], \mathbb{R}^{m}\right)$ for all $1 \leq j \leq N$.

We now define the $C^{\nu}$-network $G$ associated with $\Gamma$ as the union

$$
G=\cup_{j=1}^{N} k_{j} .
$$

The valency of each vertex $E_{i}$ is the number of edges containing $E_{i}$ and is denoted by $\gamma\left(E_{i}\right)$. We distinguish two types of vertices: the set of ramified vertices: int $E=\left\{E_{i} \in E: \gamma\left(E_{i}\right)>1\right\}$ and the set of boundary vertices: $\partial E=\left\{E_{i} \in E: \gamma\left(E_{i}\right)=1\right\}$. For shortness, we later on denote by $I_{\text {ext }}=\left\{i \in\{1 \cdots, n\}: \gamma\left(E_{i}\right)=1\right\}$ and $I_{\text {int }}=\{1 \cdots, n\} \backslash I_{\text {ext }}$. For each vertex $E_{i}$, we also denote by $N_{i}=\left\{j \in\{1, \ldots, N\}: E_{i} \in k_{j}\right\}$ the set of edges adjacent to $E_{i}$. Note that if $E_{i} \in \partial E$ then $N_{i}$ is a singleton that we write $\left\{j_{i}\right\}$.

The incidence matrix $D=\left(d_{i j}\right)_{n \times N}$ of $\Gamma$ is defined by

$$
d_{i j}=\left\{\begin{array}{lll}
1 & \text { if } & \pi_{j}\left(l_{j}\right)=E_{i} \\
-1 & \text { if } & \pi_{j}(0)=E_{i} \\
0 & \text { otherwise } &
\end{array}\right.
$$


The adjacency matrix $\mathcal{E}=\left(e_{i h}\right)_{n \times n}$ of $\Gamma$ is given by

$$
e_{i h}= \begin{cases}1 & \text { if there exists an edge } k_{s(i, h)} \text { between } E_{i} \text { and } E_{h} \\ 0 & \text { otherwise }\end{cases}
$$

For a function $u: G \rightarrow \mathbb{R}$, we set $u_{j}=u \circ \pi_{j}:\left[0, l_{j}\right] \rightarrow \mathbb{R}$, its "restriction" to the edge $k_{j}$. We further use the abbreviations:

$$
\begin{aligned}
u_{j}\left(E_{i}\right) & =u_{j}\left(\pi_{j}^{-1}\left(E_{i}\right)\right), \\
u_{j x_{j}}\left(E_{i}\right) & =\frac{d u_{j}}{d x_{j}}\left(\pi_{j}^{-1}\left(E_{i}\right)\right), \\
u_{j x_{j}^{(n)}}\left(E_{i}\right) & =\frac{d^{n} u_{j}}{d x_{j}^{n}}\left(\pi_{j}^{-1}\left(E_{i}\right)\right), n \in \mathbb{N}^{*} .
\end{aligned}
$$

For the sake of simplicity, we shall write

$$
\int_{G} u(x) d x=\sum_{j=1}^{N} \int_{0}^{l_{j}} u_{j}\left(x_{j}\right) d x_{j} .
$$

Finally, differentiations are carried out on each edge $k_{j}$ with respect to the arc length parameter $x_{j}$.

Let us now fix a $C^{4}$-network $G$ with at least one external vertex (because we want to control on the external boundary). For each edge $k_{j}$, we also fix mechanical constants $m_{j}>0$ (the mass density of the beam $k_{j}$ ) and $a_{j}=E_{j} I_{j}>0$ (the flexural rigidity of $k_{j}$ ). We consider the following operator $A$ on the Hilbert space $H=\Pi_{j=1}^{N} L^{2}\left(\left(0, l_{j}\right)\right)$, endowed with the inner product

$$
\begin{gathered}
(u, v)_{H}=\sum_{j=1}^{N} m_{j} \int_{0}^{l_{j}} u_{j}(x) v_{j}(x) d x . \\
\left\{\begin{array}{c}
D(A)=\left\{u \in H: u_{j} \in H^{4}\left(\left(0, l_{j}\right)\right) \text { satisfying }(2) \text { to }(6) \text { hereafter }\right\} \\
\forall u \in D(A): A u=\left(\frac{a_{j}}{m_{j}} u_{j x_{j}^{(4)}}\right)_{j=1}^{N} .
\end{array}\right. \\
u \text { is continuous on } G . \\
\sum_{j \in N_{i}} \frac{\partial u_{j}}{\partial \nu_{j}}\left(E_{i}\right)=0, \quad \forall i \in I_{\text {int }},
\end{gathered}
$$

where $\frac{\partial u_{j}}{\partial \nu_{j}}\left(E_{i}\right)=d_{i j} u_{j x_{j}}\left(E_{i}\right)$ means the exterior normal derivative of $u_{j}$ at $E_{i}$.

$$
\begin{aligned}
a_{j} u_{j x_{j}^{(2)}}\left(E_{i}\right) & =a_{l} u_{l x_{l}^{(2)}}\left(E_{i}\right), \quad \forall j, l \in N_{i}, \quad \forall i \in I_{i n t} . \\
\sum_{j \in N_{i}} a_{j} \frac{\partial^{3} u_{j}}{\partial \nu_{j}^{3}}\left(E_{i}\right) & =0, \quad \forall i \in I_{\text {int }} . \\
u_{j_{i}}\left(E_{i}\right)=\frac{\partial u_{j_{i}}}{\partial \nu_{j_{i}}}\left(E_{i}\right) & =0, \quad \forall i \in I_{\text {ext }} .
\end{aligned}
$$


Remark that $A$ is a nonnegative selfadjoint operator with a compact resolvant ([15], Th. 2.1) since $A$ is the Friedrichs extension of the triple $(H, V, a)$ defined by

$$
V=\left\{u \in \Pi_{j=1}^{N} H^{2}\left(\left(0, l_{j}\right)\right) \text { satisfying }(2),(3),(6)\right\}
$$

which is a Hilbert space with the inner product

$$
(u, v)_{V}=\sum_{j=1}^{N}\left(u_{j}, v_{j}\right)_{H^{2}\left(0, l_{j}\right)}
$$

when $(\cdot, \cdot)_{H^{2}\left(0, l_{j}\right)}$ is the usual $H^{2}$-inner product on $\left(0, l_{j}\right)$ and

$$
a(u, v)=\sum_{j=1}^{N} a_{j} \int_{0}^{l_{j}} u_{j x_{j}^{(2)}}\left(x_{j}\right) v_{j x_{j}^{(2)}}\left(x_{j}\right) d x_{j}
$$

The positiveness of $A$ follows from the equivalence between $a(u, u)$ and $(u, u)_{V}$ due to the fact that $G$ has (at least) one exterior vertex as the next lemma shows:

Lemma 2.1. There exists $C>0$ such that

$$
(u, u)_{V} \leq C a(u, u), \quad \forall u \in V
$$

Proof. By a standard contradiction arguments (due to the compact embedding of $V$ into $H$ ) (8) holds if we can show that $u \in V$ such that

$$
a(u, u)=0
$$

is equal to 0 .

Such a $u$ is then a polynomial of order 1 on each edge. Therefore by integration by parts and taking into account the transmission and boundary conditions $(2,3,6)$ satisfied by $u$, we get

$$
0=\sum_{j=1}^{N} \int_{0}^{l_{j}} u_{j x_{j}^{(2)}}\left(x_{j}\right) u_{j}\left(x_{j}\right) d x_{j}=-\sum_{j=1}^{N} \int_{0}^{l_{j}}\left(u_{j x_{j}}\left(x_{j}\right)\right)^{2} d x_{j}
$$

This implies that $u_{j}$ is a constant for all $j$ and by the continuity of $u$ (condition (2)), $u$ is constant on $G$. Since $u(S)=0$ for at least on external vertex $S$, we conclude that $u=0$.

For our next purposes let us denote by $\left\{\lambda_{k}\right\}_{k \in \mathbb{N}^{\star}}$ the monotone increasing sequence of the eigenvalues of $A$ repeated according to their multiplicity and for all $k \in \mathbb{N}^{\star}$, let $v^{(k)}$ be the eigenvector of $A$ associated with the eigenvalue $\lambda_{k}$. Denote further by $\left\{\tilde{\lambda}_{k}\right\}_{k \in \mathbb{N}^{\star}}$ the strictly monotone increasing sequence of the eigenvalues of $A$ not repeated according to their multiplicity. For a given eigenvalue $\tilde{\lambda}_{k}$ of $A$, we also denote by $L_{k}$ the set of $l \in \mathbb{N}^{\star}$ such that $\lambda_{l}=\tilde{\lambda}_{k}$ and by $\mathcal{N}_{k}$ the eigenspace associated with $\tilde{\lambda}_{k}$, i.e., $\mathcal{N}_{k}=\operatorname{Span}\left\{v^{(l)}\right\}_{l \in L_{k}}$. The cardinality of $L_{k}$ is clearly equal to the multiplicity of $\tilde{\lambda}_{k}$, which is uniformly bounded as the next lemma shows.

Lemma 2.2. For any eigenvalue $\lambda^{2}$ of $A$ (with $\lambda>0$ ), its multiplicity $m\left(\lambda^{2}\right)$ is less or equal to $4 N-2\left|I_{\text {ext }}\right|-1$, if $N \geq 2$, where $\left|I_{\text {ext }}\right|$ means the cardinality of the set $I_{\text {ext }}$. 
Proof. Introduce the following fundamental solutions of the fourth order derivative [15]:

$$
\left\{\begin{aligned}
e_{0}^{\lambda}(x) & =\frac{1}{2}\{\cos (\sqrt{\lambda} x)+\cosh (\sqrt{\lambda} x)\} \\
e_{1}^{\lambda}(x) & =\frac{1}{2 \sqrt{\lambda}}\{\sin (\sqrt{\lambda} x)+\sinh (\sqrt{\lambda} x)\}, \\
e_{2}^{\lambda}(x) & =\frac{1}{2 \lambda}\{-\cos (\sqrt{\lambda} x)+\cosh (\sqrt{\lambda} x)\} \\
e_{3}^{\lambda}(x) & =\frac{1}{2 \lambda^{3 / 2}}\{-\sin (\sqrt{\lambda} x)+\sinh (\sqrt{\lambda} x)\} .
\end{aligned}\right.
$$

Let $v$ be an eigenvector of $A$ associated with the eigenvalue $\lambda^{2}$. Then for all $j \in\{1, \cdots, N\}, v_{j}$ may be written

$$
v_{j}(x)=\sum_{i=0}^{3} c_{j, i} e_{i}^{\lambda}\left(\sqrt[4]{\frac{m_{j}}{a_{j}}} x\right),
$$

for some unknowns $c_{j, i}, i=0, \cdots, 3$. Since the transmission and boundary conditions (2) to (6) satisfied by $v$ are equivalent to a system of $4 N$ homogeneous (linear) equations, we get a $4 N \times 4 N$ homogeneous system of equations. Let us show that the rank of this system is at least equal to $2\left|I_{e x t}\right|+1$. Indeed for all $i \in I_{e x t}$, we can use the parametrization $\pi_{j_{i}}$ of the adjacent edge $k_{j_{i}}$ of $E_{i}$ such that $\pi_{j_{i}}(0)=E_{i}$. In this case, the boundary conditions (6) is equivalent to

$$
c_{j_{i}, 0}=c_{j_{i}, 1}=0, \quad \forall i \in I_{e x t} .
$$

This reduces our system to a $\left(4 N-2\left|I_{e x t}\right|\right) \times\left(4 N-2\left|I_{e x t}\right|\right)$ homogeneous one. For this last system, fixing one external vertex $E_{i}$ and denote by $E_{i^{\prime}}$ the other vertex of $k_{j_{i}}$ (which is an internal one except if $G$ is reduced to one interval), then the continuity of $v$ at $E_{i^{\prime}}$ furnishes a line with a nonzero element corresponding to the variable $c_{j_{i}, 2}$. This means that the rank of this system is at least one, which yields the conclusion.

Note that the above estimate is relatively rough and could be probably improved.

\section{The Petrovsky system}

Since $H, V$ and the form $a$ fulfil the hypotheses of Remark 4.4 of [27], Theorems 4.1 to 4.3 of [27] may be applied to $A$. In particular, we have the

Theorem 3.1. Let $u_{0} \in D\left(A^{s}\right), u_{1} \in D\left(A^{s-1 / 2}\right)$ and $f \in L^{1}\left(0, T ; D\left(A^{s-1 / 2}\right)\right)$, with $s \geq 1 / 2$. Then the problem

$$
\left\{\begin{array}{l}
u^{\prime \prime}(t)+A u(t)=f(t), t \in[0, T] \\
u(0)=u_{0} \\
u^{\prime}(0)=u_{1}
\end{array}\right.
$$

has a unique solution $u \in C\left([0, T], D\left(A^{s}\right)\right) \cap C^{1}\left([0, T], D\left(A^{s-1 / 2}\right)\right)$ fulfilling

$$
\|u\|_{C\left([0, T], D\left(A^{s}\right)\right)}+\|u\|_{C^{1}\left([0, T], D\left(A^{s-1 / 2}\right)\right)} \leq C\left\{\left\|u_{0}\right\|_{D\left(A^{s}\right)}+\left\|u_{1}\right\|_{D\left(A^{s-1 / 2}\right)}+\|f\|_{L^{1}\left(0, T ; D\left(A^{s-1 / 2}\right)\right)}\right\},
$$

for some constant $C>0$ independent of $u$.

In particular if $f=0$, then the energy $E(t):=\frac{1}{2}\left\{\left\|u^{\prime}(t)\right\|_{H}^{2}+a(u(t), u(t))\right\}$ is constant, for all $t \in[0, T]$ and we have

$$
E(t)=E_{0}:=\frac{1}{2}\left\{\left\|u_{1}\right\|_{H}^{2}+a\left(u_{0}, u_{0}\right)\right\}, \quad \forall t \in[0, T] .
$$

In the particular case $s=1$, the solution $u$ satisfies $u \in C([0, T], D(A))$, consequently, by the definition of $A$, $u_{j}(t)$ belongs to $H^{4}\left(\left(0, l_{j}\right)\right)$, for all $j=1, \cdots, N$. Therefore we can directly apply the classical identity with multiplier from $[17,26]$. 
Lemma 3.2. Let $u \in C([0, T], D(A)) \cap C^{1}([0, T], V)$ be the unique solution of $(10)$ and $h \in \prod_{j=1}^{N} W^{2, \infty}\left(\left(0, l_{j}\right)\right)$. Then for any $T>0$, the following identity holds:

$$
\begin{aligned}
\sum_{i=1}^{n} \sum_{j \in N_{i}} \int_{0}^{T} & \left\{h _ { j } ( E _ { i } ) d _ { i j } \left[a_{j}\left|u_{j x_{j}^{(2)}}\left(t, E_{i}\right)\right|^{2}+m_{j}\left|u_{j}^{\prime}\left(t, E_{i}\right)\right|^{2}\right.\right. \\
& \left.\left.-2 a_{j} \frac{\partial^{3} u_{j}}{\partial \nu_{j}^{3}}\left(t, E_{i}\right) \frac{\partial u_{j}}{\partial \nu_{j}}\left(t, E_{i}\right)\right]+2 a_{j} h_{j x_{j}}\left(E_{i}\right) u_{j x_{j}^{(2)}}\left(t, E_{i}\right) \frac{\partial u_{j}}{\partial \nu_{j}}\left(t, E_{i}\right)\right\} d t \\
= & \left.2 \int_{G} m(x) u^{\prime}(t, x) h(x) u_{x}(t, x) d x\right|_{0} ^{T}+\int_{0}^{T} \int_{G}\left\{h_{x}(x)\left(m(x)\left|u^{\prime}(t, x)\right|^{2}+3 a(x)\left|u_{x^{(2)}}(t, x)\right|^{2}\right)\right. \\
& \left.+2 h_{x^{(2)}}(x) a(x) u_{x^{(2)}}(t, x) u_{x}(t, x)\right\} d x d t-2 \int_{0}^{T} \int_{G} f(t, x) m(x) h(x) u_{x}(t, x) d x d t
\end{aligned}
$$

where the function a (resp. $m$ ) defined on $G$ is equal to $a_{j}\left(\right.$ resp. $\left.m_{j}\right)$ on $k_{j}$, for all $j=1, \cdots, N$.

Proof. Similar to the proof of Theorem IV.3.3 of [26] or Lemma 2.7 of [19]. We multiply the first identity of (10) by $2 m h u_{x}$ and integrate on $(0, T) \times G$. The regularity of $u$ allows to integrate by parts on each edge $k_{j}$, which leads to the conclusion.

We have voluntary kept all the boundary terms because then the above identity (12) is fully independent of the boundary conditions (6) as well as the transmission conditions (2) to (5) and is then valid for other operators. In our case, the boundary conditions (6) implies that the second, third and fourth terms of the left-hand side of (12) are equal to zero for all exterior vertices. Consequently we have the Corollary 3.3.

Corollary 3.3. Let $u \in C([0, T], D(A)) \cap C^{1}([0, T], V)$ be the unique solution of $(10)$ and $h \in \prod_{j=1}^{N} W^{2, \infty}\left(\left(0, l_{j}\right)\right)$. Then for any $T>0$, the following identity holds:

$$
\begin{aligned}
\sum_{i \in I_{\text {ext }}} \int_{0}^{T} h_{j_{i}}\left(E_{i}\right) d_{i j_{i}} a_{j_{i}}\left|u_{j_{i} x_{j_{i}}^{(2)}}\left(t, E_{i}\right)\right|^{2} d t+\sum_{i \in I_{\text {int }}} \sum_{j \in N_{i}} \int_{0}^{T}\left\{h _ { j } ( E _ { i } ) d _ { i j } \left[a_{j}\left|u_{j x_{j}^{(2)}}\left(t, E_{i}\right)\right|^{2}+m_{j}\left|u_{j}^{\prime}\left(t, E_{i}\right)\right|^{2}\right.\right. \\
\left.\left.-2 a_{j} \frac{\partial^{3} u_{j}}{\partial \nu_{j}^{3}}\left(t, E_{i}\right) \frac{\partial u_{j}}{\partial \nu_{j}}\left(t, E_{i}\right)\right]+2 a_{j} h_{j x_{j}}\left(E_{i}\right) u_{j x_{j}^{(2)}}\left(t, E_{i}\right) \frac{\partial u_{j}}{\partial \nu_{j}}\left(t, E_{i}\right)\right\} d t \\
=\left.2 \int_{G} m(x) u^{\prime}(t, x) h(x) u_{x}(t, x) d x\right|_{0} ^{T}+\int_{0}^{T} \int_{G}\left\{h_{x}(x)\left(m(x)\left|u^{\prime}(t, x)\right|^{2}+3 a(x)\left|u_{x^{(2)}}(t, x)\right|^{2}\right)\right. \\
\left.+2 h_{x^{(2)}}(x) a(x) u_{x^{(2)}}(t, x) u_{x}(t, x)\right\} d x d t-2 \int_{0}^{T} \int_{G} f(t, x) m(x) h(x) u_{x}(t, x) d x d t .
\end{aligned}
$$

The equality (13) can be applied to the solution of our system to get the so-called direct inequality (see [26], Th. IV.3.1 or [19], Th. 2.6).

Proposition 3.4. Let $u \in C([0, T], V) \cap C^{1}([0, T], H)$ be a solution of (10) with $f \in L^{1}(0, T ; V)$. Then there exists a positive constant $C$ such that for all $T>0$ it holds

$$
\sum_{i \in I_{e x t}} \int_{0}^{T}\left|u_{j_{i} x_{j_{i}}^{(2)}}\left(E_{i}, t\right)\right|^{2} d t \leq C(T+1)\left\{\left\|u_{1}\right\|_{H}^{2}+a\left(u_{0}, u_{0}\right)+\|f\|_{L^{1}(0, T ; V)}^{2}\right\} .
$$

Proof. We can split up $u=u^{(1)}+u^{(2)}$, where $u^{(1)} \in C([0, T], V) \cap C^{1}([0, T], H)$ is solution of (10) with the Cauchy data $u_{0}, u_{1}$ and $f=0$, while $u^{(2)} \in C([0, T], D(A)) \cap C^{1}([0, T], V)$ is the solution of (10) with the 
Cauchy data 0,0 and $f$. For $u^{(2)}$ using the fact that $D(A)$ is continuously embedded into $\prod_{j=1}^{N} H^{4}\left(\left(0, l_{j}\right)\right)$, the Sobolev embedding theorem and Theorem 3.1, we get

$$
\sum_{i \in I_{\text {ext }}} \int_{0}^{T}\left|u_{j_{i} x_{j_{i}}^{(2)}}^{(2)}\left(E_{i}, t\right)\right|^{2} d t \leq C T\left\|u^{(2)}\right\|_{C([0, T], D(A))}^{2} \leq C T\|f\|_{L^{1}(0, T ; V)}^{2}
$$

It then remains to prove (14) for $u^{(1)}$ that we denote by $u$ for shortness. In this case, it suffices to prove (14) for $\left(u_{0}, u_{1}\right) \in D(A) \times V$ because $D(A) \times V$ is dense in $V \times H$.

Now we apply the identity (13) with $h$ defined as follows:

i) for all edge $k_{j}$ joining interior vertices, we take $h_{j} \equiv 0$;

ii) for all edge $k_{j}$ joining an interior vertex $E_{i^{\prime}}$ to an exterior vertex $E_{i}$, take

$$
h_{j}\left(x_{j}\right)=\eta_{j}\left(x_{j}\right)\left(x_{j}-x_{j}\left(E_{i^{\prime}}\right)\right),
$$

when $\eta_{j}$ is a cut-off function such that $\eta_{j} \equiv 1$ near $E_{i}$ and $\eta_{j} \equiv 0$ near $E_{i^{\prime}}$.

Consequently, $h$ is identically equal to zero in a neighbourhood of the interior vertices and satisfies

$$
h_{j_{i}}\left(E_{i}\right) d_{i j_{i}}=l_{j_{i}}>0, \quad \forall i \in I_{e x t} .
$$

This yields

$$
\begin{aligned}
\sum_{i \in I_{\text {ext }}} \int_{0}^{T} l_{j_{i}} a_{j_{i}}\left|u_{j_{i} x_{j_{i}}^{(2)}}\left(t, E_{i}\right)\right|^{2} d t= & \left.2 \int_{G} m(x) u^{\prime}(t, x) h(x) u_{x}(t, x) d x\right|_{0} ^{T}+\int_{0}^{T} \int_{G}\left\{h _ { x } ( x ) \left(m(x)\left|u^{\prime}(t, x)\right|^{2}\right.\right. \\
& \left.\left.+3 a(x)\left|u_{x^{(2)}}(t, x)\right|^{2}\right)+2 h_{x^{(2)}}(x) a(x) u_{x^{(2)}}(t, x) u_{x}(t, x)\right\} d x d t .
\end{aligned}
$$

Using Cauchy-Schwarz inequality and the conservation of energy, we get

$$
\sum_{i \in I_{e x t}} \int_{0}^{T}\left|u_{j_{i} x_{j_{i}}^{(2)}}\left(t, E_{i}\right)\right|^{2} d t \leq C\left\{\left\|u^{\prime}\right\|_{C([0, T], H)}\|u\|_{C([0, T], V)}+T E_{0}\right\} .
$$

Owing to Theorem 3.1, we still get (14).

\section{UNIQUENESS PROPERTY}

The Hilbert uniqueness method of Lions $[19,26]$ is usually based on a inverse inequality of type

$$
\left(T-T_{0}\right) E_{0} \leq C \sum_{i \in I_{e x t}} \int_{0}^{T}\left|u_{j_{i} x_{j_{i}}^{(2)}}\left(E_{i}, t\right)\right|^{2} d t
$$

which holds for all $T>T_{0}$, for some $T_{0}>0$, where $u \in C([0, T], V) \cap C^{1}([0, T], H)$ is the unique solution of (10) with $f=0$. This guarantees that the expression

$$
\left|\left\|\left\{u_{0}, u_{1}\right\} \mid\right\|:=\left\{\sum_{i \in I_{\text {ext }}} \int_{0}^{T}\left|u_{j_{i} x_{j_{i}}^{(2)}}\left(t, E_{i}\right)\right|^{2} d t\right\}^{1 / 2},\right.
$$

is a norm on $V \times H$ (even equivalent to the norm of $V \times H$ ). To prove (16), the usual way consists in taking the identity (13) with $h_{j}=x_{j}-x_{0 j}$ and such that the boundary terms cancel except those of interest in (16) (the 
exterior only in our case). Unfortunately in our case, we remark that the sole possibility to cancel the interior boundary terms is that $h$ is equal to zero at each interior node. This means that (16) is only available for a star-shaped network. This is summarized in the

Theorem 4.1. Assume that $G$ is a star (i.e. all beams have one and one one vertex in common $E_{1}$ ), then there exists $T_{0}>0$ such that (16) holds for all $T>T_{0}$, when $u \in C([0, T], V) \cap C^{1}([0, T], H)$ is the unique solution of (10) with $f=0$.

Proof. We only need to prove (16) for $u \in C([0, T], D(A)) \cap C^{1}([0, T], V)$ solution of (10) with $f=0$ owing to a density argument.

Take $h_{j}=x_{j}-x_{j}\left(E_{1}\right)$ in the identity (13), then all the interior boundary terms are equal to zero and (13) becomes

$$
\begin{aligned}
\sum_{i \in I_{e x t}} \int_{0}^{T} l_{j_{i}} a_{j_{i}}\left|u_{j_{i} x_{j_{i}}^{(2)}}\left(t, E_{i}\right)\right|^{2} d t= & \left.2 \int_{G} m(x) u^{\prime}(t, x) h(x) u_{x}(t, x) d x\right|_{0} ^{T} \\
& +\int_{0}^{T} \int_{G}\left\{m(x)\left|u^{\prime}(t, x)\right|^{2}+3 a(x)\left|u_{x^{(2)}}(t, x)\right|^{2}\right\} d x d t .
\end{aligned}
$$

By setting

$$
\begin{aligned}
R_{0} & =\max _{i \in I_{\text {ext }}} a_{j_{i}} l_{j_{i}}, \\
X & =\left.\int_{G} m(x) u^{\prime}(t, x) h(x) u_{x}(t, x) d x\right|_{0} ^{T}, \\
Y & =\int_{0}^{T} \int_{G}\left\{m(x)\left|u^{\prime}(t, x)\right|^{2}-a(x)\left|u_{x^{(2)}}(t, x)\right|^{2}\right\} d x d t,
\end{aligned}
$$

the above identity implies that

$$
4 T E_{0}+2 X-Y \leq R_{0} \sum_{i \in I_{e x t}} \int_{0}^{T}\left|u_{j_{i} x_{j_{i}}^{(2)}}\left(t, E_{i}\right)\right|^{2} d t .
$$

But the identity (4.24) of [27] proved that

$$
Y=\left.\int_{G} m(x) u^{\prime}(t, x) u(t, x) d x\right|_{0} ^{T},
$$

consequently setting

the estimate (18) is identical with

$$
Z=\left.\int_{G} m(x) u^{\prime}(t, x)\left\{2 h(x) u_{x}(t, x)-u(t, x)\right\} d x\right|_{0} ^{T},
$$

$$
4 T E_{0}+Z \leq R_{0} \sum_{i \in I_{\text {ext }}} \int_{0}^{T}\left|u_{j_{i} x_{j_{i}}^{(2)}}\left(t, E_{i}\right)\right|^{2} d t .
$$

The conclusion now follows from the estimate

$$
|Z| \leq 4 T_{0} E_{0},
$$

which is deduced from the Cauchy-Schwarz inequality. 
Arguments similar to those from Section 5 of [19] yield equivalence between $E_{0}$ and $\left\|\mid\left\{u_{0}, u_{1}\right\}\right\| \|^{2}$ for all $T>0$, namely we have the

Corollary 4.2. Assume that $G$ is a star, then for all $T>0$, there exists a constant $C>0$ (depending on $T$ ) such that

$$
E_{0} \leq C||\left|\left\{u_{0}, u_{1}\right\}\right| \|^{2},
$$

when $u \in C([0, T], V) \cap C^{1}([0, T], H)$ is the unique solution of (10) with $f=0$.

Proof. Using the Cauchy-Schwarz inequality and the continuous embedding $D\left(A^{1 / 4}\right) \hookrightarrow \prod_{j=1}^{N} H^{1}\left(\left(0, l_{j}\right)\right)$, there exists $C_{1}>0$ such that (with the above notation)

$$
|Z| \leq C_{1} \max _{t=0, T}\left(\left\|u^{\prime}(t)\right\|_{H}\|u(t)\|_{D\left(A^{1 / 4}\right)}\right) .
$$

With the help of the inequality

$$
2 a b \leq \varepsilon a^{2}+\frac{1}{\varepsilon} b^{2},
$$

valid for all positive real numbers $a, b, \varepsilon$, and the conservation of energy, we get

$$
|Z| \leq \varepsilon E_{0}+\frac{C_{2}}{\varepsilon}\|u\|_{L^{\infty}\left(0, T ; D\left(A^{1 / 4}\right)\right)}^{2}, \quad \forall \varepsilon>0 .
$$

Inserting this estimate into (19), we obtain (compare with the estimate (3.127) in Chap. IV of [26])

$$
(4 T-\varepsilon) E_{0} \leq R_{0}\left\|\left|\left\{u_{0}, u_{1}\right\}\right|\right\|^{2}+\frac{C_{2}}{\varepsilon}\|u\|_{L^{\infty}\left(0, T ; D\left(A^{1 / 4}\right)\right)}^{2}, \quad \forall \varepsilon>0,
$$

for any $u \in C([0, T], V) \cap C^{1}([0, T], H)$ solution of (10) with $f=0$.

We now apply Theorem 5.2 of [19] with $\mathcal{Z}_{j}=\operatorname{Span} v^{(j)}$ and

$$
p(u(t))^{2}=\sum_{i \in I_{e x t}}\left|u_{j_{i} x_{j_{i}}^{(2)}}\left(E_{i}, t\right)\right|^{2} .
$$

The estimate (18) of that Theorem is guaranteed by Proposition 3.4, it then remains to check the estimate (17) which in our cases is equivalent to

$$
E_{0} \leq C_{3} \int_{0}^{T^{\star}} p(u(t))^{2} d t
$$

for all $u \in C\left(\left[0, T^{\star}\right], V\right) \cap C^{1}\left(\left[0, T^{\star}\right], H\right)$ solution of $(10)$ with $f=0$ and $u_{0}, u_{1}$ orthogonal to $v^{(j)}, j=1, \cdots, k-1$, for some $k \in \mathbb{N}^{\star}$ and some $T^{\star}>0$ (the constant $C_{3}$ depending on $k$ and $T^{\star}$ ). Since by Theorem 5.2 of [19], we then have (20) for all $T>T^{\star}$, it suffices to check (22) for all $T^{\star}>0$ if $k$ is large enough.

But the spectral Theorem directly yields

$$
\|u\|_{L^{\infty}\left(0, T^{\star} ; D\left(A^{1 / 4}\right)\right)}^{2} \leq 2 \lambda_{k}^{-1 / 2} E_{0} .
$$

Therefore the estimate (21) and the above one lead to

$$
\left(4 T^{\star}-\varepsilon-\frac{2 C_{2}}{\varepsilon \lambda_{k}^{1 / 2}}\right) E_{0} \leq R_{0}\left\|||\left\{u_{0}, u_{1}\right\} \mid\right\|^{2}, \quad \forall \varepsilon>0 .
$$


Consequently, (22) holds if we choose $\varepsilon=3 T^{\star}$ and $k$ large enough such that $\frac{2 C_{2}}{\varepsilon \lambda_{k}^{1 / 2}}<T^{\star}$.

Since we want to treat other networks than the stars, we are looking for sufficient conditions insuring that \|\|$\cdot \|$ is a norm on $V \times H$ and which is relatively practical to be checked for a given example. This will be done with the help of Ingham's inequality [3]. Therefore we suppose that the spectrum of $A$ satisfies the condition

$$
\underline{\lim }_{k \rightarrow \infty}\left(\sqrt{\tilde{\lambda}_{k+1}}-\sqrt{\tilde{\lambda}_{k}}\right)=+\infty
$$

Theorem 4.3. If $A$ satisfies (24), then for all $T>0,\|\| \cdot \|$ is a norm on $V \times H$ if and only if (25) hereafter holds.

$$
\text { For all } k \in \mathbb{N}^{\star} \text {, any } v \in \mathcal{N}_{k} \backslash\{0\} \text { satisfies } \sum_{i \in I_{e x t}}\left|v_{j_{i} x_{j_{i}}^{(2)}}\left(E_{i}\right)\right|^{2}>0 \text {. }
$$

Proof. Let us first assume that $\left\{u_{0}, u_{1}\right\}$ is actually in $D(A) \times V$ and let $u \in C([0, T], D(A)) \cap C^{1}([0, T], V)$ be the unique solution of (10) with $f=0$. Then the spectral theorem allows to write

$$
u(t, \cdot)=\sum_{k \in \mathbb{N}^{\star}}\left(u_{0 k} \cos \left(t \sqrt{\lambda_{k}}\right)+u_{1 k} \frac{\sin \left(t \sqrt{\lambda_{k}}\right)}{\sqrt{\lambda_{k}}}\right) v^{(k)},
$$

where the $u_{i k}$ 's are defined by

$$
u_{i}=\sum_{k \in \mathbb{N}^{\star}} u_{i k} v^{(k)}, i=0,1 .
$$

The above identity may be written equivalently

$$
u(t, \cdot)=\sum_{k \in \mathbb{N}^{\star}}\left\{\cos \left(t \sqrt{\tilde{\lambda}_{k}}\right)\left(\sum_{l \in L_{k}} u_{0 l} v^{(l)}\right)+\frac{\sin \left(t \sqrt{\tilde{\lambda}_{k}}\right)}{\sqrt{\tilde{\lambda}_{k}}}\left(\sum_{l \in L_{k}} u_{1 l} v^{(l)}\right)\right\} .
$$

Since each $v^{(k)}$ belongs to $D(A)$ and due to the inclusion $D(A) \hookrightarrow \prod_{j=1}^{N} H^{4}\left(\left(0, l_{j}\right)\right)$ and the Sobolev embedding theorem we get

$$
\left|v_{j_{i} x_{j_{i}}^{(2)}}^{(k)}\left(E_{i}\right)\right| \leq C\left\|v^{(k)}\right\|_{H^{4}\left(\left(0, l_{j_{i}}\right)\right)} \leq C\left\|v^{(k)}\right\|_{D(A)} \leq C \lambda_{k}, \quad \forall i \in I_{e x t} .
$$

This implies that for all $i \in I_{\text {ext }}$ the series

$$
\sum_{k \in \mathbb{N}^{\star}}\left\{\left|\cos \left(t \sqrt{\tilde{\lambda}_{k}}\right)\right|^{2}\left|\sum_{l \in L_{k}} u_{0 l} v_{j_{i} x_{j_{i}}^{(2)}}^{(l)}\left(E_{i}\right)\right|^{2}+\left|\frac{\sin \left(t \sqrt{\tilde{\lambda}_{k}}\right)}{\sqrt{\tilde{\lambda}_{k}}}\right|^{2}\left|\sum_{l \in L_{k}} u_{1 l} v_{j_{i} x_{j_{i}}^{(2)}}^{(l)}\left(E_{i}\right)\right|^{2}\right\}
$$

is convergent because it is bounded by $\left\|u_{0}\right\|_{D(A)}^{2}+\left\|u_{1}\right\|_{V}^{2}$. 
With the help of Ingham's inequalities [18], we now prove that this implies that for all $T>0$ and all $i \in I_{\text {ext }}$, we have

$$
u_{j_{i} x_{j_{i}}^{(2)}}\left(t, E_{i}\right)=\sum_{k \in \mathbb{N}^{*}}\left\{\cos \left(t \sqrt{\tilde{\lambda}_{k}}\right)\left(\sum_{l \in L_{k}} u_{0 l} v_{j_{i} x_{j_{i}}^{(2)}}^{(l)}\left(E_{i}\right)\right)+\frac{\sin \left(t \sqrt{\tilde{\lambda}_{k}}\right)}{\sqrt{\tilde{\lambda}_{k}}}\left(\sum_{l \in L_{k}} u_{1 l} v_{j_{i} x_{j_{i}}^{(2)}}^{(l)}\left(E_{i}\right)\right)\right\}
$$

this identity being understood as an identity in $L^{2}((0, T))$. And furthermore that there exist positive constants $C_{1}, C_{2}$ (depending on $T$ ) such that

$$
\begin{aligned}
C_{1} \sum_{k \in \mathbb{N}^{\star}}\left\{\left|\sum_{l \in L_{k}} u_{0 l} v_{j_{i} x_{j_{i}}^{(2)}}^{(l)}\left(E_{i}\right)\right|^{2}\right. & \left.+\frac{1}{\tilde{\lambda}_{k}}\left|\sum_{l \in L_{k}} u_{1 l} v_{j_{i} x_{j_{i}}^{(2)}}^{(l)}\left(E_{i}\right)\right|^{2}\right\} \leq \int_{0}^{T}\left|u_{j_{i} x_{j_{i}}^{(2)}}\left(t, E_{i}\right)\right|^{2} d t \\
& \leq C_{2} \sum_{k \in \mathbb{N}^{\star}}\left\{\left|\sum_{l \in L_{k}} u_{0 l} v_{j_{i} x_{j_{i}}^{(2)}}^{(l)}\left(E_{i}\right)\right|^{2}+\frac{1}{\tilde{\lambda}_{k}}\left|\sum_{l \in L_{k}} u_{1 l} v_{j_{i} x_{j_{i}}^{(2)}}^{(l)}\left(E_{i}\right)\right|^{2}\right\} .
\end{aligned}
$$

First we apply the version of Ingham's inequalities of Theorem 2.1 of [3] to

$$
v_{i}(t):=\sum_{k \in \mathbb{N}^{*}}\left\{\cos \left(t \sqrt{\tilde{\lambda}_{k}}\right)\left(\sum_{l \in L_{k}} u_{0 l} v_{j_{i} x_{j_{i}}^{(2)}}^{(l)}\left(E_{i}\right)\right)+\frac{\sin \left(t \sqrt{\tilde{\lambda}_{k}}\right)}{\sqrt{\tilde{\lambda}_{k}}}\left(\sum_{l \in L_{k}} u_{1 l} v_{j_{i} x_{j_{i}}^{(2)}}^{(l)}\left(E_{i}\right)\right)\right\} .
$$

More precisely applying Theorem 2.1 of [3] to the truncated series and passing to the limit, we get the existence of $C_{3}, C_{4}>0$ (depending on the parameter $\gamma$ hereafter) such that

$$
\begin{aligned}
C_{3} T \sum_{k \in \mathbb{N}^{\star}}\left\{\left|\sum_{l \in L_{k}} u_{0 l} v_{j_{i} x_{j_{i}}^{(2)}}^{(l)}\left(E_{i}\right)\right|^{2}\right. & \left.+\frac{1}{\tilde{\lambda}_{k}}\left|\sum_{l \in L_{k}} u_{1 l} v_{j_{i} x_{j_{i}}^{(2)}}^{(l)}\left(E_{i}\right)\right|^{2}\right\} \leq \int_{0}^{T}\left|v_{i}(t)\right|^{2} d t \\
& \leq C_{4} T \sum_{k \in \mathbb{N}^{\star}}\left\{\left|\sum_{l \in L_{k}} u_{0 l} v_{j_{i} x_{j_{i}}^{(2)}}^{(l)}\left(E_{i}\right)\right|^{2}+\frac{1}{\tilde{\lambda}_{k}}\left|\sum_{l \in L_{k}} u_{1 l} v_{j_{i} x_{j_{i}}^{(2)}}^{(l)}\left(E_{i}\right)\right|^{2}\right\},
\end{aligned}
$$

for all $T>0$ satisfying the assumption (2.1) of [3] which, in our setting, is reduced to

$$
\left\{\begin{array}{l}
\underline{\lim }_{k \rightarrow \infty}\left(\sqrt{\tilde{\lambda}_{k+1}}-\sqrt{\tilde{\lambda}_{k}}\right)>\gamma>0 \\
T>\frac{2 \pi}{\gamma}
\end{array}\right.
$$

Consequently the assumption (24) implies that (28) holds for all $T>0$.

Secondly if we consider the sequence

$$
w^{(K)}:=\sum_{k \leq K}\left\{\cos \left(t \sqrt{\tilde{\lambda}_{k}}\right)\left(\sum_{l \in L_{k}} u_{0 l} v^{(l)}\right)+\frac{\sin \left(t \sqrt{\tilde{\lambda}_{k}}\right)}{\sqrt{\tilde{\lambda}_{k}}}\left(\sum_{l \in L_{k}} u_{1 l} v^{(l)}\right)\right\}, K \in \mathbb{N}^{\star} .
$$

By Theorem 3.1, it is a Cauchy sequence in $C([0, T], D(A))$ and due to the embedding $D(A) \hookrightarrow \prod_{j=1}^{N} C^{2}\left(\left[0, l_{j}\right]\right)$, we deduce that

$$
w_{j_{i} x_{j_{i}}^{(2)}}^{(K)}\left(\cdot, E_{i}\right) \rightarrow u_{j_{i} x_{j_{i}}^{(2)}}\left(\cdot, E_{i}\right) \text { in } C([0, T]) \text {, as } K \rightarrow \infty,
$$


and thus also in $L^{2}((0, T))$. This fact and the estimates (28) lead to (26) and (27).

Summing the estimates $(27)$ on $i \in I_{\text {ext }}$, we get the equivalence

$$
\begin{aligned}
C_{1} \sum_{k \in \mathbb{N}^{\star}}\left\{\sum_{i \in I_{\text {ext }}}\left|\sum_{l \in L_{k}} u_{0 l} v_{j_{i} x_{j_{i}}^{(2)}}^{(l)}\left(E_{i}\right)\right|^{2}\right. & \left.+\frac{1}{\tilde{\lambda}_{k}} \sum_{i \in I_{\text {ext }}}\left|\sum_{l \in L_{k}} u_{1 l} v_{j_{i} x_{j_{i}}^{(2)}}^{(l)}\left(E_{i}\right)\right|^{2}\right\} \leq\left|\left\|\left\{u_{0}, u_{1}\right\}\right\|\right|^{2} \\
& \leq C_{2} \sum_{k \in \mathbb{N}^{\star}}\left\{\sum_{i \in I_{\text {ext }}}\left|\sum_{l \in L_{k}} u_{0 l} v_{j_{i} x_{j_{i}}^{(2)}}^{(l)}\left(E_{i}\right)\right|^{2}+\frac{1}{\tilde{\lambda}_{k}} \sum_{i \in I_{\text {ext }}}\left|\sum_{l \in L_{k}} u_{1 l} v_{j_{i} x_{j_{i}}^{(2)}}^{(l)}\left(E_{i}\right)\right|^{2}\right\} .
\end{aligned}
$$

The density of $D(A) \times V$ into $V \times H$ and Proposition 3.4 (with $f=0$ ) implies that (29) also holds for $\left\{u_{0}, u_{1}\right\}$ in $V \times H$.

From this equivalence, we see that ||$\left|\left\{u_{0}, u_{1}\right\}\right| \|=0$ if and only if

$$
\sum_{l \in L_{k}} u_{0 l} v_{j_{i} x_{j_{i}}^{(2)}}^{(l)}\left(E_{i}\right)=\sum_{l \in L_{k}} u_{1 l} v_{j_{i} x_{j_{i}}^{(2)}}^{(l)}\left(E_{i}\right)=0, \quad \forall i \in I_{\text {ext }}, \quad \forall k \in \mathbb{N}^{\star}
$$

Therefore if (25) holds we get

$$
u_{0 k}=u_{1 k}=0, \quad \forall k \in \mathbb{N}^{\star}
$$

Indeed for a fixed $k \in \mathbb{N}^{\star}$, consider

$$
\begin{aligned}
& w^{0}=\sum_{l \in L_{k}} u_{0 l} v^{(l)}, \\
& w^{1}=\sum_{l \in L_{k}} u_{1 l} v^{(l)} .
\end{aligned}
$$

Clearly $w^{0}, w^{1}$ belong to $\mathcal{N}_{k}$ and satisfy

$$
w_{j_{i} x_{j_{i}}^{(2)}}^{0}\left(E_{i}\right)=w_{j_{i} x_{j_{i}}^{(2)}}^{1}\left(E_{i}\right)=0, \quad \forall i \in I_{e x t} .
$$

Consequently the assumption (25) implies that $w^{0}=w^{1}=0$. As the eigenvectors $v^{(l)}$ are linearly independent, we get (30).

In conclusion, as (30) implies that $u_{0}=u_{1}=0$, we have shown that (25) guarantees that $\||\cdot|\|$ is a norm on $V \times H$.

Conversely if (25) does not hold, then there exists (at least) one eigenvalue $\tilde{\lambda}_{k}$ and a nonzero eigenvector $v \in \mathcal{N}_{k}$ such that

$$
v_{j_{i} x_{j_{i}}^{(2)}}\left(E_{i}\right)=0, \quad \forall i \in I_{\text {ext }} .
$$

Therefore $u(t)=v \cos \left(t \sqrt{\tilde{\lambda}_{k}}\right)$ is a solution of (10) with $f=0$ and initial data $\{v, 0\} \neq 0$. From (29), we deduce that ||$\{v, 0\} \mid \|=0$. Consequently, $|\| \cdot|||$ is not a norm on $V \times H$.

The assumption (24) is justified by the analysis of some examples where it is satisfied (see Sect. 7 for some examples). Note that the assumption (24) is satisfied for an interval. It is also satisfied for star-shaped networks with edges of length 1 and coefficients $a_{j}=m_{j}$; indeed using the method from [15], one can show that the spectrum $\sigma(A)$ of $A$ is given by

$$
\sigma(A)=\left\{\tilde{\lambda}_{1, k}^{2}\right\}_{k \in \mathbb{N}^{\star}} \cup\left\{\tilde{\lambda}_{2, k}^{2}\right\}_{k \in \mathbb{N}^{*}},
$$


where the $\tilde{\lambda}_{1, k}$ are the positive roots of

$$
\tanh (\sqrt{\lambda})+\tan (\sqrt{\lambda})=0
$$

the multiplicity of $\tilde{\lambda}_{1, k}^{2}$ being 1 ; while the $\tilde{\lambda}_{2, k}$ are the positive roots of

$$
\tanh (\sqrt{\lambda})-\tan (\sqrt{\lambda})=0
$$

the multiplicity of $\tilde{\lambda}_{2, k}^{2}$ being $N-1$ ( $N$ is the number of edges of the network). The assumption (24) is then satisfied because

$$
\begin{aligned}
& \sqrt{\tilde{\lambda}_{1, k}}+\frac{\pi}{4}-k \pi \rightarrow 0, \text { as } k \rightarrow \infty \\
& \sqrt{\tilde{\lambda}_{2, k}}-\frac{\pi}{4}-k \pi \rightarrow 0, \text { as } k \rightarrow \infty
\end{aligned}
$$

From Theorem 4.3 and Corollary 4.2, the condition (25) holds for these networks (this can also be checked by the explicit knowledge of the eigenvectors).

For arbitrary networks, by Theorem 4.2 of [15], we know that

$$
\lambda_{k}=c^{4}\left(k+f_{k}\right)^{4}, \quad \forall k \in \mathbb{N}^{\star},
$$

for some positive constant $c$ and a bounded sequence $\left\{f_{k}\right\}_{k \in \mathbb{N}^{*}}$ i.e., there exists $C>0$ such that

$$
\left|f_{k}\right| \leq C, \quad \forall k \in \mathbb{N}^{\star}
$$

In particular (31) implies that

$$
\lim _{k \rightarrow \infty} \frac{\lambda_{k}}{k^{4}}=c^{4},
$$

and furthermore

$$
\sqrt{\lambda_{k+1}}-\sqrt{\lambda_{k}}=c^{2}\left(2 k \alpha_{k}+\beta_{k}\right),
$$

where we have set

$$
\begin{aligned}
\alpha_{k} & =1+f_{k+1}-f_{k} \geq 0, \\
\beta_{k} & =1+2 f_{k+1}+f_{k+1}^{2}-f_{k}^{2} \geq 0 .
\end{aligned}
$$

Accordingly if

$$
\varliminf_{k \rightarrow \infty} \alpha_{k}>0
$$

then (24) holds, on the other hand if

$$
\varliminf_{k \rightarrow \infty} \alpha_{k}=0
$$

then

$$
\varliminf_{k \rightarrow \infty} \beta_{k}=0,
$$


and we need to analyze the asymptotic behaviour of $k \alpha_{k}$ : Either

$$
\varliminf_{k \rightarrow \infty} k \alpha_{k}=\alpha>0
$$

or

$$
\varliminf_{k \rightarrow \infty} k \alpha_{k}=0
$$

must hold. In the first case, we then have

$$
\varliminf_{k \rightarrow \infty}\left(\sqrt{\lambda_{k+1}}-\sqrt{\lambda_{k}}\right)=2 c^{2} \alpha
$$

which implies that the equivalence in Theorem 4.3 only holds for $T$ large enough. In the second alternative, we have

$$
\varliminf_{k \rightarrow \infty}\left(\sqrt{\lambda_{k+1}}-\sqrt{\lambda_{k}}\right)=0
$$

and from the examples given in [3], we may expect that $|\|\cdot \mid\|$ is not a norm on $V \times H$.

In Examples 7.2 to 7.4 that we will analyze, we will see that the set $\left\{\lambda_{k}^{1 / 4}\right\}_{k \in \mathbb{N}^{\star}}$ is quasi-periodic, i.e.,

$$
f_{m k+j} \rightarrow \gamma_{j}-j, \quad \forall j=1, \cdots, m, \text { as } k \rightarrow \infty,
$$

for some $m \in \mathbb{N}^{\star}$ with the properties that

$$
\left\{\begin{array}{l}
\gamma_{j+1}-\gamma_{j}>0, \quad \forall j=1, \cdots, m-1 \\
\gamma_{1}+m-\gamma_{m}>0
\end{array}\right.
$$

This implies that (34) holds and the above considerations then yield the

Lemma 4.4. If the operator A satisfies (38) and (39), then its large eigenvalues are simple and it satisfies (24).

\section{WEAK SOlUtions OF THE WAVE EQUATION}

We transpose Proposition 3.4 to get the

Theorem 5.1. For all $u_{0} \in H, u_{1} \in V^{\prime}, w_{i} \in L^{2}((0, T)), i \in I_{\text {ext }}$, there exist unique $u \in L^{\infty}\left(0, T ; V^{\prime}\right)$, $\left\{\psi_{1}, \psi_{0}\right\} \in V^{\prime} \times H$, which are solutions of

$$
\begin{aligned}
\int_{0}^{T}<u(t), f(t)>_{V^{\prime}-V} d t & +<\psi_{1}, \varphi_{0}>_{V^{\prime}-V}-\left(\psi_{0}, \varphi_{1}\right)_{H}=<u_{1}, \varphi(0)>_{V^{\prime}-V}-\left(u_{0}, \varphi^{\prime}(0)\right)_{H} \\
& -\sum_{i \in I_{e x t}} \int_{0}^{T} w_{i} \varphi_{j_{i} x_{j_{i}}^{(2)}}\left(t, E_{i}\right) d t, \quad \forall f \in L^{1}(0, T ; V),\left\{\varphi_{0}, \varphi_{1}\right\} \in V \times H,
\end{aligned}
$$

where $\varphi$ is the unique solution of

$$
\left\{\begin{array}{l}
\varphi \in C([0, T], V) \cap C^{1}([0, T], H) \\
\varphi^{\prime \prime}(t)+A \varphi(t)=f(t), t \in[0, T] \\
\varphi(T)=\varphi_{0}, \varphi^{\prime}(T)=\varphi_{1}
\end{array}\right.
$$


Formally, the solutions $u,\left\{\psi_{1}, \psi_{0}\right\}$ of (40) satisfy

$$
\left\{\begin{array}{l}
u_{j}^{\prime \prime}\left(t, x_{j}\right)+a_{j} \frac{\partial^{4} u_{j}}{\partial x_{j}^{4}}=0, \text { on }(0, T) \times\left(0, l_{j}\right), \quad \forall j=1, \cdots, N \\
u_{j}(t, \cdot) \text { satisfies }(2) \text { to }(5) \\
u_{j_{i}}\left(E_{i}\right)=0, \quad \forall i \in I_{e x t}, \\
\frac{\partial u_{j_{i}}}{\partial \nu_{j_{i}}}\left(E_{i}\right)=a_{j_{i}}^{-1} w_{i}, \quad \forall i \in I_{e x t}, \\
u(0)=u_{0}, u^{\prime}(0)=u_{1}
\end{array}\right.
$$

and the final conditions

$$
u(T)=\psi_{0}, u^{\prime}(T)=\psi_{1}
$$

This is the case for more regular data as we show below. In the case of the above Theorem, we shall actually prove that $u$ is more regular in order to give a meaning to (43). This is made in the spirit of Theorem 2.9 of [19] or Theorem 5.3 of [27]. In order to satisfy (43), the minimal regularity for $u$ seems to be

$$
u \in C([0, T], H) \cap C^{1}\left([0, T], V^{\prime}\right) .
$$

This motivates the following definition.

Definition 5.2. We say that $u$ is a weak solution of (42) if $u$ has the regularity (44) and $u,\left\{u^{\prime}(T), u(T)\right\}$ are the unique solutions of (40).

First the next trace lifting result will be useful.

Lemma 5.3. Let $w_{i} \in \mathcal{D}((0, T)), i \in I_{\text {ext }}$. Then there exists $v \in \mathcal{D}(0, T) \prod_{j=1}^{N} C^{\infty}\left(\left[0, l_{j}\right]\right)$ fulfilling (2) to (5) and

$$
\begin{aligned}
v_{j_{i}}\left(E_{i}\right) & =0, \quad \forall i \in I_{e x t} \\
\frac{\partial v_{j_{i}}}{\partial \nu_{j_{i}}}\left(E_{i}\right) & =a_{j_{i}}^{-1} w_{i}, \quad \forall i \in I_{e x t}
\end{aligned}
$$

Proof. We let the reader check that $v$ defined herebelow satisfies the desired boundary conditions.

i) for all edge $k_{j}$ joining interior vertices, we take $v_{j} \equiv 0$;

ii) for all edge $k_{j}$ joining an interior vertex $E_{i^{\prime}}$ to an exterior vertex $E_{i}$, take

$$
v_{j}\left(x_{j}\right)=\eta_{j}\left(x_{j}\right)\left(x_{j}-x_{j}\left(E_{i}\right)\right) d_{i j} a_{j}^{-1} w_{i}
$$

when $\eta_{j}$ is a cut-off function such that $\eta_{j} \equiv 1$ near $E_{i}$ and $\eta_{j} \equiv 0$ near $E_{i^{\prime}}$

Theorem 5.4. Let $u \in L^{\infty}\left(0, T ; V^{\prime}\right),\left\{\psi_{1}, \psi_{0}\right\} \in V^{\prime} \times H$ be the unique solutions of (40) with data $u_{0} \in V$, $u_{1} \in H$ and $w_{i} \in \mathcal{D}((0, T)), i \in I_{\text {ext }}$. Then

$$
u \in C\left([0, T], \prod_{j=1}^{N} H^{2}\left(\left(0, l_{j}\right)\right)\right) \cap C^{1}([0, T], H)
$$

and satisfies (42) and (43). 
Proof. We proceed as in Theorem 5.3 of [27] with the necessary adaptations. Let us fix $v \in \mathcal{D}(0, T) \prod_{j=1}^{N} C^{\infty}$ $\left(\left[0, l_{j}\right]\right)$ obtained in Lemma 5.3 and set

$$
f_{j}=v_{j}^{\prime \prime}+a_{j} v_{j x_{j}^{(4)}}, \quad \forall 1 \leq j \leq N
$$

Since $f \in L^{2}(0, T ; H)$, Lemma I.3.4 of [26] guarantees the existence of a unique solution $\psi \in C([0, T], V) \cap$ $C^{1}([0, T], H) \cap H^{2}\left(0, T ; V^{\prime}\right)$ of

$$
\left\{\begin{array}{l}
<\psi^{\prime \prime}(t), w>_{V^{\prime}-V}+a(\psi(t), w) \\
=-\int_{G} f(t, x) w(x) d x, \text { a.e. } t \in[0, T], \quad \forall w \in V \\
\psi(0)=u_{0}, \psi^{\prime}(0)=u_{1}
\end{array}\right.
$$

From the definition of $v$ and the above problem solved by $\psi$, we easily check that

$$
u=\psi+v
$$

satisfies (42) and has the regularity (47).

Let us now show that $u$ is the unique solution of (40) when $\psi_{0}=u(T), \psi_{1}=u^{\prime}(T)$.

By Theorem 4.2 of [27], it suffices to check (40) for $\varphi \in C([0, T], D(A)) \cap C^{1}([0, T], V) \cap C^{2}([0, T], H)$. Since $u \in H^{2}\left(0, T ; V^{\prime}\right)$, the integrations by parts over $(0, T)$ are allowed. Taking into account the initial conditions satisfied by $\varphi$ and $u$ and the regularities of $v$ and $\psi$, we get

$$
\begin{aligned}
\int_{0}^{T}\left\langle u(t), \varphi^{\prime \prime}(t)\right. & +A \varphi(t)\rangle_{V^{\prime}-V} d t-\left(u(T), \varphi_{1}\right)_{H}+\left\langle u^{\prime}(T), \varphi_{0}\right\rangle_{V^{\prime}-V}=\left\langle u_{1}, \varphi(0)\right\rangle_{V^{\prime}-V}-\left(u_{0}, \varphi^{\prime}(0)\right)_{H} \\
& +\int_{0}^{T}\left\{\left\langle\psi^{\prime \prime}(t), \varphi(t)\right\rangle_{V^{\prime}-V}+\left\langle v^{\prime \prime}(t), \varphi(t)\right\rangle_{V^{\prime}-V}+a(\psi(t), \varphi(t))+(v(t), A \varphi(t))_{H}\right\} d t
\end{aligned}
$$

By integration by parts and using the boundary and transmission conditions satisfied by $v$ and $\varphi$, the term $(v(t), A \varphi(t))_{H}$ is transformed into

$$
(v(t), A \varphi(t))_{H}=\int_{G} a(x) v_{x^{(4)}}(t, x) \varphi(t, x) d x-\sum_{i \in I_{e x t}} \int_{0}^{T} w_{i} \varphi_{j_{i} x_{j_{i}}^{(2)}}\left(t, E_{i}\right) d t
$$

Inserting this identity into (50), using the definition of $f$ and (48), we see that the right-hand side of (50) is exactly equal to the right-hand side of (40). This is the desired identity.

Combining the two above theorems and density arguments, we deduce that the unique solutions $u,\left\{\psi_{1}, \psi_{0}\right\}$ of (40) satisfy $u \in C\left([0, T], V^{\prime}\right)$ and $u(T)=\psi_{0}$. But no regularity for the derivative $u^{\prime}$ is available. In order to get it, we use the usual trick of reduction of order (see paragraph 5 of [27]).

Theorem 5.5. Let $u \in L^{\infty}\left(0, T ; V^{\prime}\right),\left\{\psi_{1}, \psi_{0}\right\} \in V^{\prime} \times H$ be the unique solutions of (40) with data $u_{0} \in H$, $u_{1} \in V^{\prime}$ and $w_{i} \in L^{2}((0, T)), i \in I_{\text {ext }}$. Then $u$ is a weak solution of (42).

Proof. We argue as at the end of paragraph 5 of [27]: we first reduce the wave equation to the first order equation

$$
\left\{\begin{array}{l}
U^{\prime}+B U=F \\
U(0)=U_{0}
\end{array}\right.
$$


where $B$ is an operator from $\mathcal{H}=V \times H$ into itself defined by $D(B)=D(A) \times V$ and for all $U=(u, v) \in D(B)$, $B U=(-v, A u)$. Using Lemma 5.4 of [27] and Proposition 3.4, we directly conclude that if $U=(u, v) \in$ $C([0, T], \mathcal{H})$ is the unique solution of $(51)$ with $U_{0}=\left(u_{0}, u_{1}\right) \in \mathcal{H}$ and $F \in L^{1}(0, T ; D(B))$, then $u$ satisfies

$$
\sum_{i \in I_{e x t}} \int_{0}^{T}\left|u_{j_{i} x_{j_{i}}^{(2)}}\left(E_{i}, t\right)\right|^{2} d t \leq C(T+1)\left\{\left\|u_{1}\right\|_{H}^{2}+a\left(u_{0}, u_{0}\right)+\|f\|_{L^{1}(0, T ; V)}^{2}\right\} .
$$

By transposition and density, we arrive at the conclusion.

\section{The Hilbert uniqueness method}

The application of the Hilbert uniqueness method of Lions [26] is now quite standard: firstly, by Proposition 3.4, for $\left\{\varphi_{0}, \varphi_{1}\right\} \in V \times H$, there exists a unique solution $\varphi \in C([0, T], V) \cap C^{1}([0, T], H)$ of (10) with $f=0$, satisfying (14). Secondly, consider $\psi \in L^{\infty}\left(0, T, V^{\prime}\right),\left\{\chi_{1}, \chi_{0}\right\} \in V^{\prime} \times H$, the unique solutions of

$$
\begin{aligned}
\int_{0}^{T}\langle\psi(t), g(t)\rangle_{V^{\prime}-V} d t & -\left\langle\chi_{1}, \eta_{0}\right\rangle_{V^{\prime}-V}+\left(\chi_{0}, \eta_{1}\right)_{H} \\
= & -\sum_{i \in I_{\text {ext }}} \int_{0}^{T} \varphi_{j_{i} x_{j_{i}}^{(2)}}\left(t, E_{i}\right) \eta_{j_{i} x_{j_{i}}^{(2)}}\left(t, E_{i}\right) d t, \quad \forall g \in L^{1}(0, T ; V),\left\{\eta_{0}, \eta_{1}\right\} \in V \times H,
\end{aligned}
$$

where $\eta$ is the unique solution of

$$
\left\{\begin{array}{l}
\eta \in C([0, T], V) \cap C^{1}([0, T], H) \\
\eta^{\prime \prime}(t)+A \eta(t)=g(t), t \in[0, T] \\
\eta(0)=\eta_{0}, \eta^{\prime}(0)=\eta_{1}
\end{array}\right.
$$

Its existence comes from Theorem 5.1 with time reversed; moreover, Theorem 5.5 guarantees that $\psi \in C([0, T], H) \cap$ $C^{1}\left([0, T], V^{\prime}\right)$ and gives a meaning to the initial conditions

$$
\psi(0)=\chi_{0}, \psi^{\prime}(0)=\chi_{1} .
$$

Accordingly, the next operator is well-defined

$$
\Lambda: V \times H \rightarrow V^{\prime} \times H:\left\{\varphi_{0}, \varphi_{1}\right\} \rightarrow\left\{\chi_{1},-\chi_{0}\right\}
$$

but unfortunately it is not an isomorphism in general. Indeed the identity (52) with $\eta=\varphi$ yields

$$
\left\langle\Lambda\left\{\varphi_{0}, \varphi_{1}\right\},\left\{\varphi_{0}, \varphi_{1}\right\}\right\rangle=\sum_{i \in I_{e x t}} \int_{0}^{T}\left|\varphi_{j_{i} x_{j_{i}}^{(2)}}\left(t, E_{i}\right)\right|^{2} d t
$$

Therefore, $\Lambda$ will be an isomorphism if and only if the semi-norm $\|\cdot\| \mid \|$ is a norm on $V \times H$. By Theorem 4.3, this is the case if (24) and (25) hold. In this case, we define $F$ as the closure of $V \times H$ for this new norm. Furthermore by Proposition 3.4 we have the continuous and dense embedding

$$
V \times H \hookrightarrow F .
$$

Consequently, by density, the identity (54) implies that $\Lambda$ is an isomorphism from $F$ into $F^{\prime}$. This leads to the main result of this section: 
Theorem 6.1. If $G$ is a star or if (24) and (25) hold, then for all $\left\{u_{1},-u_{0}\right\} \in F^{\prime}$, there exist $w_{i} \in L^{2}((0, T))$, $i \in I_{\text {ext }}$ such that the weak solution $u \in C([0, T], H) \cap C^{1}\left([0, T], V^{\prime}\right)$ of the wave equation (42) satisfies

$$
u(T)=u^{\prime}(T)=0 .
$$

If $G$ is a star, we further have $F=V \times H$.

Proof. First start with $\left\{u_{1},-u_{0}\right\} \in \Lambda(V \times H)$ (dense subset of $\left.F^{\prime}\right)$, then denote by $\left\{\varphi_{0}, \varphi_{1}\right\} \in V \times H$ the unique element such that

$$
\Lambda\left\{\varphi_{0}, \varphi_{1}\right\}=\left\{u_{1},-u_{0}\right\}
$$

We take the solution $\varphi$ of (10) with $f=0$ and then the solution $\psi \in C([0, T], H) \cap C^{1}\left([0, T], V^{\prime}\right)$ of $(52)$. In this case, the conclusion follows with $u=\psi, w_{i}=\varphi_{j_{i} x_{j_{i}}^{(2)}}\left(t, E_{i}\right)$, for all $i \in I_{e x t}$, because of the reversibility of the wave equation and Proposition 3.4. Furthermore we remark by Theorem 5.5 and the isomorphic property of $\Lambda$ that there exists $C>0$ (which depends on $T$ ) such that

$$
\|u\|_{C([0, T], H)}+\left\|u^{\prime}\right\|_{C\left([0, T], V^{\prime}\right)} \leq C\left\|\left\{u_{1},-u_{0}\right\}\right\|_{F^{\prime}} .
$$

This last estimate and a density argument allow to get the conclusion for any $\left\{u_{1},-u_{0}\right\} \in F^{\prime}$.

If $G$ is a star, Theorem 4.1 clearly implies that $F=V \times H$.

Remark 6.2. In view of Theorem 4.3 the space $F$ is equal to $D\left(A^{s}\right) \times D\left(A^{s-1 / 2}\right)$, for some $s \leq 1 / 2$ if and only if there exists $C>0$ such that

$$
\sum_{i \in I_{\text {ext }}}\left|\sum_{l \in L_{k}} x_{l} v_{j_{i} x_{j_{i}}^{(2)}}^{(l)}\left(E_{i}\right)\right|^{2} \geq C \lambda_{k}^{2 s} \sum_{l \in L_{k}} x_{l}^{2}, \quad \forall\left(x_{l}\right)_{l \in L_{k}} \in \mathbb{R}^{\left|L_{k}\right|}, \quad \forall k \in \mathbb{N}^{\star},
$$

which is not easy to prove in general. Obviously by Theorem 4.1 this estimate holds with $s=1 / 2$ for star-shaped networks. For general networks, a deeper spectral analysis of $A$ is necessary. At this stage it is not quite clear if the above estimate holds or not. Nevertheless, even if $F^{\prime}$ is not known we shall show that the sufficient condition (25) to have exact controllability is also necessary.

Remark 6.3. Note that the whole machinery extends to other kinds of transmission conditions like those from $[9,15]$.

\section{NeCESSARY CONDItion FOR THE EXACT CONTROLlabiLity AND EXAMPLES}

In this section, we shall show that (25) is a necessary condition in order to have exact controllability. We further give four examples of networks (not star-shaped): three for which we have exact controllability and one for which we do not have exact controllability. For this last one, we choose a network with a circuit as in ([23], $\S$ II.5.2).

We now remark that the exact controllability at time $T>0$ of our problem by Dirichlet control on the external boundary with the help of HUM is equivalent to say that the continuous mapping

$$
C_{T}: L^{2}((0, T))^{\left|I_{e x t}\right|} \longrightarrow F^{\prime}:\left(w_{i}\right)_{i \in I_{e x t}} \longmapsto\left\{u^{\prime}(0), u(0)\right\},
$$

where $u \in C([0, T], H) \cap C^{1}\left([0, T], V^{\prime}\right)$ is the weak solution of $(42)$ with $u(T)=u^{\prime}(T)=0$, is surjective.

From (40), we directly see that

$$
C_{T}^{*}\left(\left\{-\eta_{0}, \eta_{1}\right\}\right)=\left(\eta_{j_{i} x_{j_{i}}^{(2)}}\left(\cdot, E_{i}\right)\right)_{i \in I_{e} x t}
$$


where $\eta$ is the unique solution of (53).

Assume now that (25) does not hold, this means that there exists (at least) one eigenvector $v \neq 0$ of $A$ associated with the eigenvalue $\lambda$ satisfying $v_{j_{i} x_{j_{i}}^{(2)}}\left(E_{i}\right)=0$, for all $i \in I_{\text {ext }}$. Therefore $\eta(t)=v \cos (t \sqrt{\lambda})$ is a solution of (53) with initial data $\{v, 0\}$. This implies that the pair $\{v, 0\} \in D(A) \times V \hookrightarrow F$ belongs to $\operatorname{ker} C_{T}^{*}$ since

$$
\eta_{j_{i} x_{j_{i}}^{(2)}}\left(t, E_{i}\right)=v_{j_{i} x_{j_{i}}^{(2)}}\left(E_{i}\right) \cos (t \sqrt{\lambda})=0, \quad \forall i \in I_{e x t} .
$$

Therefore $C_{T}$ is not surjective which proves the

Corollary 7.1. If (25) does not hold, then our problem (42) is not exactly controllable at any time $T>0$ (in the sense of Th. 6.1).

In view of Theorem 6.1 to prove the exact controllability, it suffices to check the spectral conditions (24) and (25). This is the method we used on the next three examples.

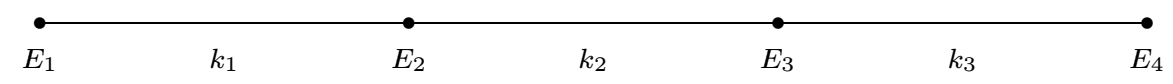

Figure 1. Three serially connected beams.

Example 7.2. Take the network $G$ with three serially connected beams $k_{1}, k_{2}, k_{3}$ of length 1 [10] i.e. $k_{1} \cap k_{2}=$ $\left\{E_{2}\right\}, k_{2} \cap k_{3}=\left\{E_{3}\right\}, k_{1} \cap k_{3}=\emptyset$ (see Fig. 1). Take $a_{1}=m_{1}=1, a_{2}=m_{2}=2$ and $a_{3}=m_{3}=4$. Using the techniques from [15] and with the help of a symbolic language, we see that $\lambda^{2} \neq 0$ is an eigenvalue of $A$ if and only if $d(\lambda)=0$, where

$$
\begin{aligned}
d(\lambda)= & -364+36 \cos (2 \sqrt{\lambda})+\{-103 \cos \sqrt{\lambda}+35 \cos (3 \sqrt{\lambda})\} \cosh \sqrt{\lambda}+36\{1+\cos (2 \sqrt{\lambda})\} \cosh (2 \sqrt{\lambda}) \\
& +\{35 \cos \sqrt{\lambda}+289 \cos (3 \sqrt{\lambda})\} \cosh (3 \sqrt{\lambda}) .
\end{aligned}
$$

To check the assumption (24), we need to study the asymptotic behaviour of the zeroes of the function $d$. Since $d(\lambda)=0$ if and only if $\tilde{d}(\lambda)=0$, where $\tilde{d}(\lambda)=\frac{d(\lambda)}{\cosh (3 \sqrt{\lambda})}$, we are reduced to the analysis of the zeroes of $\tilde{d}$. But it may be written

$$
\tilde{d}(\lambda)=q(\cos \sqrt{\lambda})+r(\lambda)
$$

where $q(\cos \sqrt{\lambda})=35 \cos \sqrt{\lambda}+289 \cos (3 \sqrt{\lambda})$ and $r$ is the remainder which satisfies

$$
|r(\lambda)| \leq C e^{-\sqrt{\lambda}}, \quad \forall \lambda>0,
$$

for some $C>0$. Therefore a zero $\lambda$ of $\tilde{d}$ satisfies

$$
|q(\cos \sqrt{\lambda})| \leq C e^{-\sqrt{\lambda}}
$$

which means that $\cos \sqrt{\lambda}$ is close to a zero of $q$ if $\lambda$ is large. The assumption (24) will follow from this fact and from the periodicity of the set of solutions of $q(\cos \sqrt{\lambda})=0$ if $q$ has only simple roots.

We are now looking for the zeroes of $q$. By a usual trigometric formula, we see that

$$
q(x)=4 x\left[289 x^{2}-16 \times 13\right] .
$$




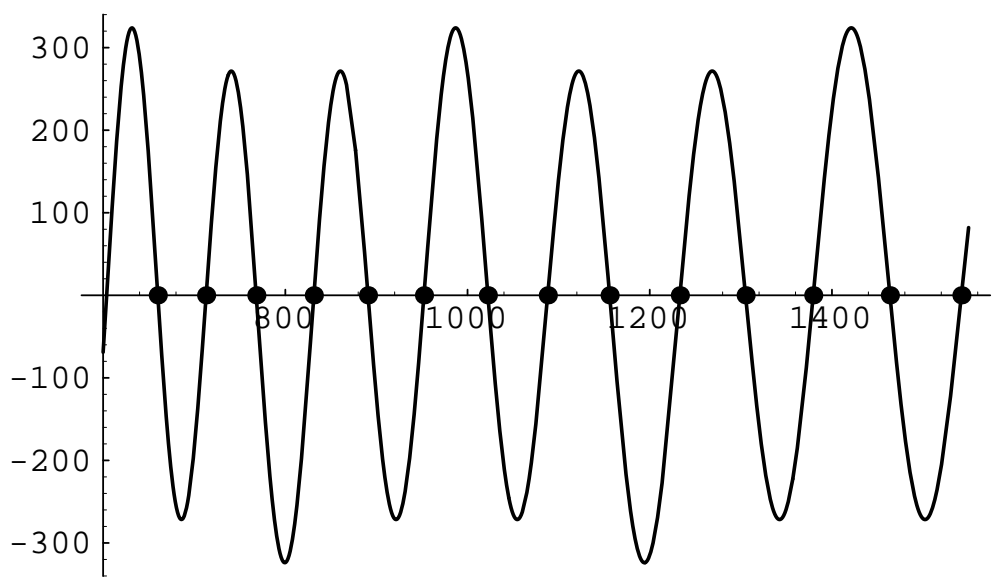

Figure 2. The zeroes of the function $\tilde{d}$ for 3 beams.

Its zeroes are $0, a_{+}=\frac{4 \sqrt{13}}{\sqrt{289}}<1$ and $a_{-}=-a_{+}$. Denote by $\omega_{j}, j=1, \cdots, 6$ the angles in $] 0,2 \pi[$ such that $\cos \omega_{j}=0, a_{-}$or $a_{+}$and enumerated in increasing order. The above considerations imply that the spectrum $\left\{\lambda_{k}\right\}_{k \in \mathbb{N}^{\star}}$ of $A$ satisfies

$$
\lambda_{6 k+j}-\left(\omega_{j}+2 k \pi\right)^{4} \rightarrow 0, \text { as } k \rightarrow \infty, \quad \forall j=1, \cdots, 6 .
$$

This means that the assumptions (38) and (39) are satisfied with $m=6$ and by Lemma 4.4 (24) holds. This asymptotic behaviour is illustrated in Figure 2 where we have plotted the function $\tilde{d}(\lambda)$ in an interval of length $9 \pi^{2}$ as well as the points $\left(\omega_{j}+2 k \pi\right)^{2}$ in the same interval. We see that the roots of $d$ are very close to these points.

Moreover by direct calculations (as in [15]), we can show that there exist no eigenvectors $v$ satisfying (2) to (6) and

$$
v_{1 x_{1}^{(2)}}\left(E_{1}\right)=0, v_{3 x_{3}^{(2)}}\left(E_{4}\right)=0,
$$

when $E_{1}$ (resp. $E_{4}$ ) is the external node of $G$ at $k_{1}$ (resp. $k_{3}$ ). Consequently this network satisfies the spectral condition (25) and is then exactly controllable at any time $T>0$.

Example 7.3. Take the network $G$ with four serially connected beams $k_{1}, k_{2}, k_{3}, k_{4}$ of length 1 i.e. $k_{1} \cap k_{2}=$ $\left\{E_{2}\right\}, k_{2} \cap k_{3}=\left\{E_{3}\right\}, k_{3} \cap k_{4}=\left\{E_{4}\right\}, k_{1} \cap k_{4}=\emptyset$ (see Fig. 3).

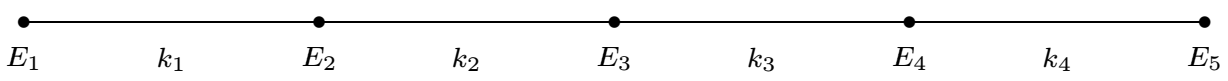

FiguRE 3. Four serially connected beams.

Take $a_{1}=m_{1}=1, a_{2}=m_{2}=2, a_{3}=m_{3}=4$ and $a_{4}=m_{4}=8$. As before, we see that $\lambda^{2} \neq 0$ is an eigenvalue of $A$ if and only if $d(\lambda)=0$, where

$$
\begin{aligned}
d(\lambda)= & 7111-684 \cos (2 \sqrt{\lambda})-307 \cos (4 \sqrt{\lambda})+\{3960 \cos \sqrt{\lambda}-1368 \cos (3 \sqrt{\lambda})\} \cosh \sqrt{\lambda} \\
& +\{-684+1008 \cos (2 \sqrt{\lambda})-612 \cos (4 \sqrt{\lambda})\} \cosh (2 \sqrt{\lambda})+\{1368 \cos \sqrt{\lambda}-1224 \cos (3 \sqrt{\lambda})\} \cosh (3 \sqrt{\lambda}) \\
& +\{-307-612 \cos (2 \sqrt{\lambda})-4913 \cos (4 \sqrt{\lambda})\} \cosh (4 \sqrt{\lambda}) .
\end{aligned}
$$


Here the "dominant" term is the term of $\cosh (4 \sqrt{\lambda})$, and then we are looking for the zeroes of its factor, namely the solutions of

$$
q(\cos (2 \sqrt{\lambda}))=-307-612 \cos (2 \sqrt{\lambda})-4913 \cos (4 \sqrt{\lambda})=0 .
$$

Since $q$ has four different roots in ] - 1, 1[, we check as in the first example that the assumptions (38) and (39) are satisfied with $m=8$; and by Lemma 4.4 (24) is satisfied (see Fig. 4 for an illustration).

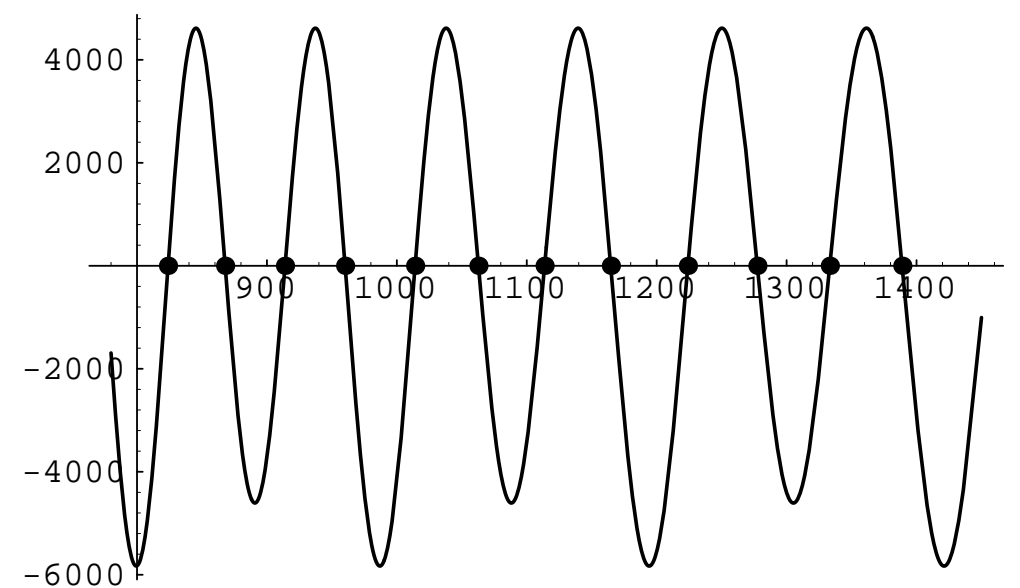

Figure 4. The zeroes of the function $\tilde{d}$ for 4 beams.

We can also show that this network satisfies the spectral condition (25) (this is checked by showing that any $v \in \mathcal{N}_{k}$ satisfying $v_{1 x_{1}^{(2)}}\left(E_{1}\right)=0, v_{4 x_{4}^{(2)}}\left(E_{4}\right)=0$, is equal to zero) and is then exactly controllable at any time $T>0$.

The next example is concerned with a network different from a star and with noncollinear beams.

Example 7.4. Take the network $G$ with four beams $k_{1}, k_{2}, k_{3}, k_{4}$ of length 1 "in T" defined by (see Fig. 5)

$$
\begin{aligned}
& k_{1}=(0,1) \times\{0\}, k_{2}=(1,2) \times\{0\}, \\
& k_{3}=\{2\} \times(0,1), k_{4}=\{2\} \times(-1,0) .
\end{aligned}
$$

Take $a_{1}=m_{1}=1, a_{j}=m_{j}=2, j=2,3,4$. As before, we can show that $\lambda^{2} \neq 0$ is an eigenvalue of $A$ if and only if $d(\lambda)=0$, where

$$
\begin{aligned}
d(\lambda)= & \sum_{j=0}^{3}\left\{p_{j}(\cos (\sqrt{\lambda}), \sin (\sqrt{\lambda})) \cosh (j \sqrt{\lambda})+q_{j}(\cos (\sqrt{\lambda}), \sin (\sqrt{\lambda})) \sinh (j \sqrt{\lambda})\right\} \\
& +\{-96 \sin (2 \sqrt{\lambda})+153 \sin (4 \sqrt{\lambda})\} \cosh (4 \sqrt{\lambda})-\{57+114 \cos (2 \sqrt{\lambda})+153 \cos (4 \sqrt{\lambda})\} \sinh (4 \sqrt{\lambda}),
\end{aligned}
$$

where $p_{j}, q_{j}, j=0, \cdots, 3$ are polynomials of order at most 4 (explicitly known but not given for shortness). Here the "dominant" terms are those of $\cosh (4 \sqrt{\lambda})$ and of $\sinh (4 \sqrt{\lambda})$, then we are looking for the solutions $\lambda$ of

$$
-96 \sin (2 \sqrt{\lambda})+153 \sin (4 \sqrt{\lambda})-\{57+114 \cos (2 \sqrt{\lambda})+153 \cos (4 \sqrt{\lambda})\}=0
$$




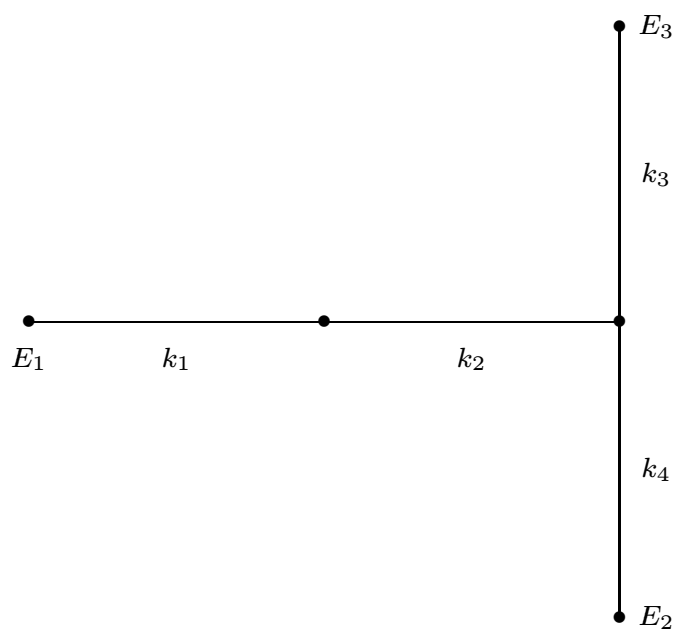

Figure 5. Four beams in T.

Due to usual trigonometric rules, these roots are related to the zeroes in $[-1,1]$ of the polynomial

$$
q(y)=\left(57+114 y+153\left(2 y^{2}-1\right)\right)^{2}-\left(1-y^{2}\right)(306 y-96)^{2},
$$

plotted in Figure 6. Denote by $y_{j}, j=1, \cdots, 4$ its roots in increasing order. Note that $y_{2}=0$ and approximate values of the other ones are

$$
y_{1} \approx-0.976781, y_{3} \approx 0.363402, y_{4} \approx 0.554556 .
$$

We then check that the (positive) solutions $\lambda$ of (55) are given by

$$
\lambda=\left(\omega_{j} / 2+k \pi\right)^{2}, k \in \mathbb{N}, j=1, \cdots, 4,
$$

when $\omega_{2}=\pi / 2$ and $\omega_{j}, j=1,3,4$ are such that $\cos \omega_{j}=y_{j}$ and

$$
\sin \omega_{1}<0, \sin \omega_{3}<0, \sin \omega_{4}>0 .
$$

They approximately are equal to

$$
\omega_{1} \approx 3.35750, \quad \omega_{3} \approx 5.08430, \quad \omega_{4} \approx 0.982967 .
$$

Because $\cosh (4 \sqrt{\lambda})$ and $\sinh (4 \sqrt{\lambda})$ are equivalent to $e^{4 \sqrt{\lambda}}$ at $+\infty$, we deduce that

$$
\lambda_{4 k+j}-\left(\omega_{j} / 2+k \pi\right)^{4} \rightarrow 0, \text { as } k \rightarrow \infty, \quad \forall j=1, \cdots, 4 .
$$

Therefore (38) and (39) are satisfied with $m=4$ and by Lemma 4.4, (24) holds. The above asymptotic behaviour is illustrated in Figure 7 where we have plotted the function $\tilde{d}(\lambda)=d(\lambda) / \cosh (4 \sqrt{\lambda})$ in an interval of length $9 \pi^{2}$ as well as the points $\left(\omega_{j} / 2+k \pi\right)^{2}$ in the same interval.

As before we can show that this network satisfies the spectral condition (25) and is then exactly controllable at any time $T>0$. 


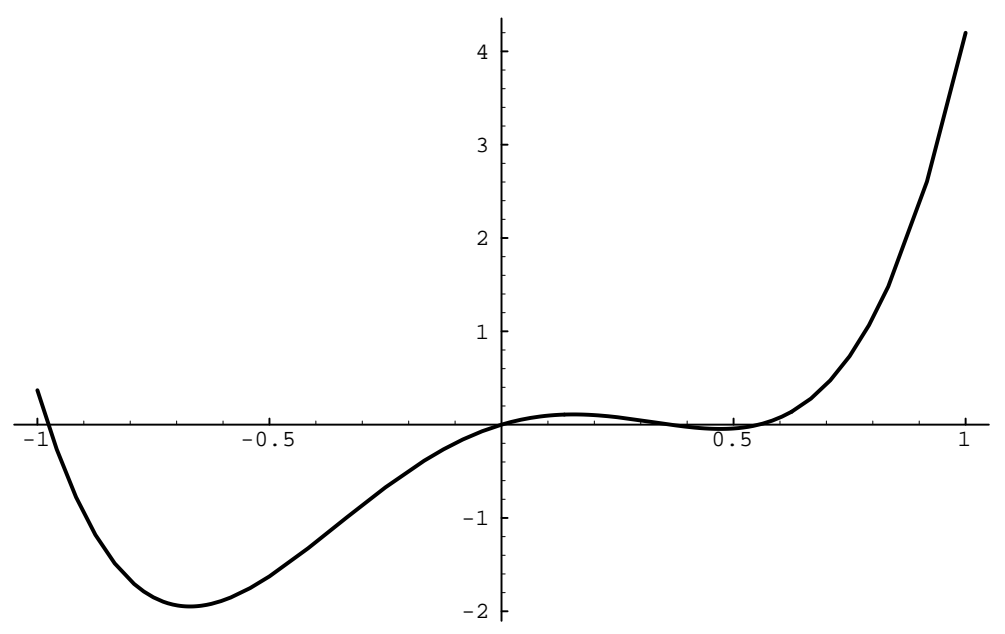

Figure 6 . The polynomial $q$.

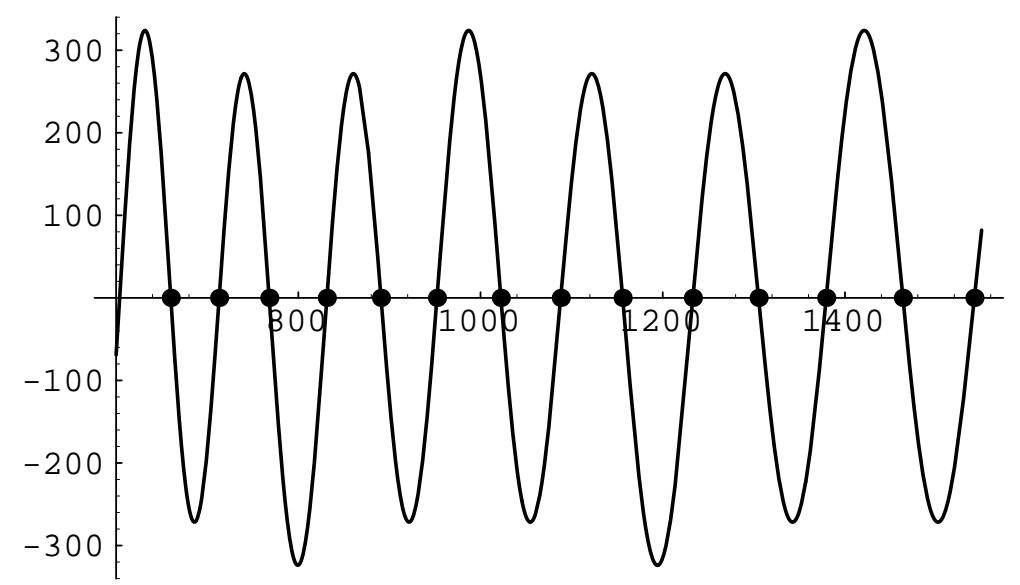

Figure 7 . The zeroes of the function $\tilde{d}$ for 4 beams in T.

Example 7.5. Let us define $G \subset \mathbb{R}^{2}$ by (see Fig. 8)

$$
G=\cup_{i=-3}^{3} k_{i}
$$

where

$$
\begin{aligned}
& k_{0}=\left(0, l_{0}\right) \times\{0\}, k_{1}=\left\{l_{0}\right\} \times\left(0, l_{1}\right), \\
& k_{2}=\left\{l_{1}\right\} \times\left(l_{1}, l_{1}+l_{2}\right), k_{3}=\left\{l_{0}+l_{1}\right\} \times\left(0, l_{1}\right), \\
& k_{-l}=\left\{\left(x_{1},-x_{2}\right):\left(x_{1}, x_{2}\right) \in k_{l}\right\}, l=1,2,3 .
\end{aligned}
$$

The exterior edge of $G$ is reduced to the point $E_{1}=(0,0)$. Take $a_{-j}=a_{j}$ and $m_{-j}=m_{j}$, for all $j=1,2,3$ and $a_{0}, m_{0}$ arbitrary. 


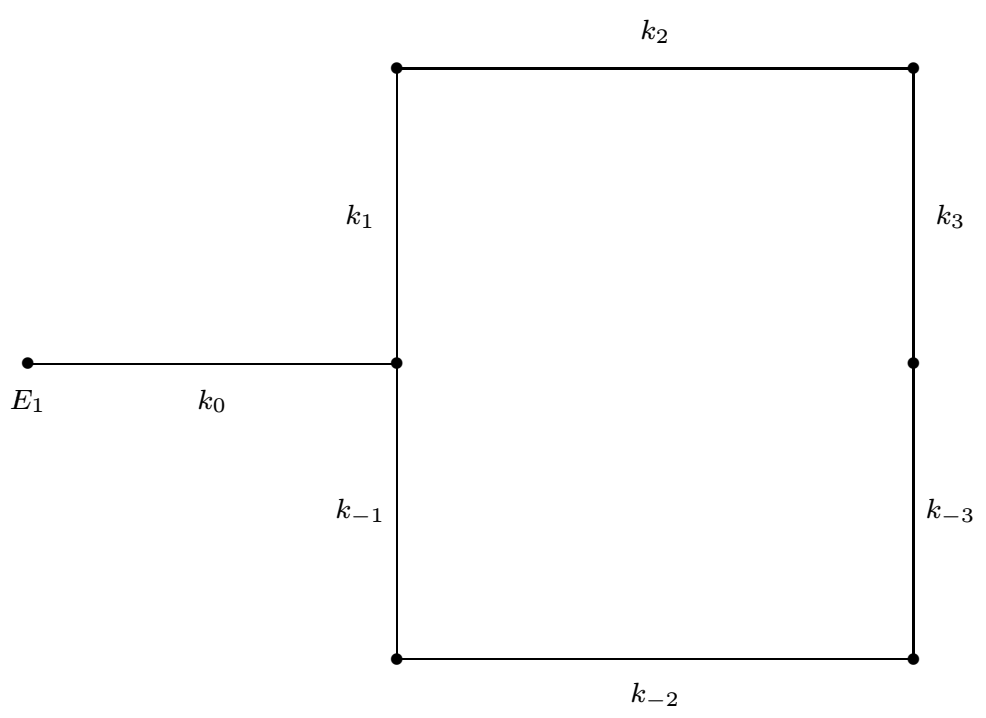

FiguRE 8. A network with a circuit.

According to Corollary 7.1 (see also [23]), the lack of controllability comes from the existence of a special eigenvector $w \neq 0$ of our operator $A$ on $G$ of eigenvalue $\lambda>0$ fulfilling then

$$
a_{j} w_{j x_{j}^{(4)}}=\lambda m_{j} w_{j} \text { in } k_{j}, \quad \forall j \in\{-3,-2,-1,0,1,2,3\}
$$

and the boundary and transmission conditions (2) to (6) and the supplementary condition

$$
w_{1 x_{1}^{(2)}}\left(E_{1}\right)=0 .
$$

Indeed, let us consider the network $\tilde{G}=\cup_{j=1}^{3} k_{j}$ and the operator $\tilde{A}$ on $\tilde{G}$ with Dirichlet boundary conditions on its exterior boundary (corresponding to the vertices of $k_{1}$ and $k_{3}$ included in the line $x_{2}=0$ ). Take $\tilde{w}=\left(w_{j}\right)_{j=1,2,3}$ one eigenvector $\neq 0$ of $\tilde{A}$ of eigenvalue $\lambda>0$ (in other words, $\tilde{w}$ satisfies (56) for $j=1,2,3$, transmission conditions at the interior nodes of $\tilde{G}$ and Dirichlet boundary conditions at exterior nodes of $\tilde{G}$ ). We now define $w$ on the whole of $G$ by symmetry:

$$
w_{0} \equiv 0, w_{-l}\left(x_{1},-x_{2}\right)=w_{l}\left(x_{1}, x_{2}\right), \quad \forall\left(x_{1}, x_{2}\right) \in k_{l}, l=1,2,3 .
$$

From the inclusion $\tilde{w} \in D(\tilde{A})$, we readily check that $w \in D(A)$ and satisfies (56). The property (57) is immediate since $w_{0} \equiv 0$ in $k_{0}$.

This means that the network $G$ does not satisfy the spectral condition (25) and is then not exactly controllable by external boundary action.

Remark 7.6. Since the goal of the Examples 7.2 to 7.4 was to illustrate the general theory, the parameters $a_{j}$ and $m_{j}$ were chosen as simple as possible in order to avoid too complicated calculations, but in order to have examples which cannot be reduced to star-shaped networks for which we always have exact controllability (for instance in Ex. 7.2, if we take $a_{1}=a_{2}, m_{1}=m_{2}$, then the example reduces to a star-shaped network with one edge of length 2 and one of length 1 ).

We thank Dr. I. Cattiaux-Huillard for her help in the numerical computations needed in the examples from Section 7. We also thank the referees for valuable remarks and comments on the first version of that paper. 


\section{REFERENCES}

[1] F. Ali Mehmeti, A characterisation of generalized $C^{\infty}$ notion on nets. Int. Eq. and Operator Theory 9 (1986) 753-766.

[2] F. Ali Mehmeti, Regular solutions of transmission and interaction problems for wave equations. Math. Meth. Appl. Sci. 11 (1989) 665-685.

[3] J.M. Ball and M. Slemrod, Nonharmonic Fourier series and the stabilization of distributed semi-linear control systems. Comm. Pure Appl. Math. 32 (1979) 555-587.

[4] J. von Below, A characteristic equation associated to an eigenvalue problem on $c^{2}$-networks. Linear Alg. Appl. 71 (1985) 309-325.

[5] J. von Below, Classical solvability of linear parabolic equations on networks. J. Diff. Eq. 72 (1988) 316-337.

[6] J. von Below, Sturm-Liouville eigenvalue problems on networks. Math. Meth. Appl. Sci. 10 (1988) 383-395.

[7] J. von Below, Parabolic Network Equations. Habilitation Thesis, Eberhard-Karls-Universität Tübingen (1993).

[8] J. von Below and S. Nicaise, Dynamical interface transition with diffusion in ramified media. Comm. Partial Diff. Eq. 21 (1996) 255-279.

[9] A. Borovskikh, R. Mustafokulov, K. Lazarev and Yu. Pokornyi, A class of fourth-order differential equations on a spatial net. Doklady Math. 52 (1995) 433-435.

[10] G. Chen, M. Delfour, A. Krall and G. Payre, Modelling, stabilization and control of serially connected beams. SIAM J. Control and Opt. 25 (1987) 526-546.

[11] G. Chen, S. Krantz, D. Russell, C. Wayne, H. West and M. Coleman, Analysis, design, and behavior of dissipative joints for coupled beams. SIAM J. Appl. Math. 49 (1989) 1665-1693.

[12] G. Chen and J. Zhou, The wave propagation method for the analysis of boudary stabilization in vibrating structures. SIAM J. Appl. Math. 50 (1990) 1254-1283.

[13] P.G. Ciarlet, H. Le Dret and R. Nzengwa, Junctions between three-dimension and two-dimensional linearly elastic structures. J. Math. Pures Appl. 68 (1989) 261-295.

[14] F. Conrad, Stabilization of vibrating beams by a specific feedback, A.V. Balakrishnan and J.P. Zolésio Eds., Stabilization of flexible structures, Opt. Software Inc. (1988) 36-51.

[15] B. Dekoninck and S. Nicaise, The eigenvalue problem for networks of beams. Preprint LIMAV 96-9, University of Valenciennes, Linear Alg. Appl. (submitted).

[16] P. Grisvard, Elliptic problems in nonsmooth domains. Monographs and Studies in Mathematics 21 (Pitman, Boston, 1985).

[17] P. Grisvard, Contrôlabilité exacte des solutions de l'équation des ondes en présence de singularités. J. Math. Pures Appl. 68 (1989) 215-259.

[18] A.E. Ingham, Some trigonometrical inequalities with applications in the theory of series. Math. Z. 41 (1936) 367-369.

[19] V. Komornik, Exact controllability and stabilization. The multiplier method. RMA 36 Masson, Paris (1994).

[20] J.E. Lagnese, Modeling and controllability of plate-beam systems. J. Math. Systems, Estimation and Control. 5 (1995) $141-187$.

[21] J.E. Lagnese, G. Leugering and E.J.P.G. Schmidt, Modeling of dynamic networks of thin thermoelastic beams. Math. Meth. Appl. Sci. 16 (1993) 327-358.

[22] J.E. Lagnese, G. Leugering and E.J.P.G. Schmidt, Control of planar networks of Timoshenko beams. SIAM J. Cont. Opt. 31 (1993) $780-811$.

[23] J.E. Lagnese, G. Leugering and E.J.P.G. Schmidt, Modeling, analysis and control of dynamic elastic multi-link structures, Birkhäuser, Boston (1994).

[24] H. Le Dret, Problèmes variationnels dans les multi-domaines. Modélisation des jonctions et applications. RMA 19, Masson, Paris (1991).

[25] G. Leugering and E.J.P.G. Schmidt, On the control of networks of vibrating strings and beams, in Proc. of the 28th IEEE Conference on Decision and Control, Vol. 3, IEEE (1989) 2287-2290.

[26] J.-L. Lions, Contrôlabilité exacte, perturbations et stabilisation de systèmes distribués. Tome 1, RMA 8, Masson, Paris (1988).

[27] S. Nicaise, Exact controllability of a pluridimensional coupled problem. Rev. Math. Univ. Complutense Madrid 5 (1992) 91-135.

[28] S. Nicaise, About the Lamé system in a polygonal or a polyhedral domain and a coupled problem between the Lamé system and the plate equation II: Exact controllability. Ann. Scuola Normale Sup. Pisa, Series IV 20 (1993) 163-191.

[29] S. Nicaise, Boundary exact controllability of interface problems with singularities I: Addition of the coefficients of singularities. SIAM J. Contr. Opt. 34 (1996) 1512-1533.

[30] S. Nicaise, Boundary exact controllability of interface problems with singularities II: Addition of internal controls. SIAM J. Contr. Opt. 35 (1997) 585-603.

[31] J.P. Puel and E. Zuazua, Exact controllability for a model of multidimensional flexible structure. Proc. Royal Soc. Edinburgh 123 A (1993) 323-344.

[32] E.J.P.G. Schmidt, On the modelling and exact controllability of networks of vibrating strings. SIAM J. Contr. Opt. 30 (1992) $229-245$. 